\title{
Terbium-induced phase transitions and weak ferromagnetism in multiferroic bismuth ferrite ceramics
}

Vladimir Koval $^{1}$, Ivan Skorvanek ${ }^{2}$, Juraj Durisin ${ }^{1}$, Giuseppe Viola ${ }^{3,4}$, Alexandra Kovalcikova ${ }^{1}$, Peter Svec jr. ${ }^{5}$, Karel Saksl ${ }^{1}$, and Haixue Yan $^{4}$

${ }^{1}$ Institute of Materials Research, Slovak Academy of Sciences, Kosice, Slovakia

${ }^{2}$ Institute of Experimental Physics, Slovak Academy of Sciences, Kosice, Slovakia

${ }^{3}$ Institute of Materials Physics and Engineering, Torino, Italy

${ }^{4}$ School of Engineering and Materials Science, Queen Mary, University of London, London, United Kingdom, Mile End Road, E1 4NS

${ }^{5}$ Institute of Physics, Slovak Academy of Sciences, Bratislava, Slovakia

\begin{abstract}
Partial substitution of isovalent rare-earths for bismuth is one of the most effective ways to develop room temperature $\mathrm{BiFeO}_{3}$-based multiferroic materials with high resistivity and strong magnetoelectric coupling. However, their structures and properties are composition and processing sensitive, with underlying mechanisms still far from complete understanding. Here we report on the structural, thermal and magnetic properties of polycrystalline $\mathrm{Bi}_{1-\mathrm{x}} \mathrm{Tb}_{\mathrm{x}} \mathrm{FeO}_{3}(0$ $\leq \mathrm{x} \leq 0.30$ ) dense ceramics prepared by spark plasma sintering (SPS). X-ray diffraction study reveals that increasing terbium content induces a structural transformation from the parental rhombohedral $(R 3 c)$ polar phase to an orthorhombic (Pnma) non-polar phase at $\mathrm{x} \approx 0.20-0.25$. Complementary Raman and energy-loss near-edge structure (ELNES) spectroscopy studies indicate that the transition proceeds by the progressive loss of the $\mathrm{Bi}-\mathrm{O}$ hybridization. A suppression of the long-range ferroelectric ordering upon $\mathrm{Tb}$ substitution and loss ferroelectricity at $\mathrm{x} \geq 0.25$ was also confirmed by differential scanning calorimetry. Highsensitive magnetic measurements show that the introduction of a small amount of $\mathrm{Tb}^{3+}$ ions at the A- sites of the perovskite structure gives rise to the occurrence of the spontaneous magnetization at room temperature. The reduced degree of the $\mathrm{Fe} 3 d-4 p$ orbital mixing and the weaker Fe $3 d$ - O $2 p$ hybridization, revealed by the ELNES and X-ray near-edge absorption fine structure (NEXAFS) analyses, suggest that the substitution-induced changes in the electronic structure are responsible for the enhanced magnetization in $\mathrm{Tb}$-doped $\mathrm{BiFeO}_{3}$. Among biphasic $(R 3 c+P n m a)$ compositions with the ferroelectric order, the $\mathrm{Bi}_{0.8} \mathrm{~Tb}_{0.2} \mathrm{FeO}_{3}$
\end{abstract}


compound shows the highest values of the remanent magnetization $\left(\mathrm{M}_{\mathrm{r}} \approx 0.26 \mathrm{emu} / \mathrm{g}\right)$, which makes this material a potential candidate for magnetoelectric applications.

\section{Introduction}

Bismuth ferrite, $\mathrm{BiFeO}_{3}$, belongs to a family of multiferroic materials that show at least two ferroic states (ferromagnetism or anti-ferromagnetism, ferroelectricity or anti-ferroelectricity, ferroelasticity or antiferroelasticity) within the same phase at the same temperature and pressure. $\left[{ }^{1}\right]$ Since the revival of interest in magnetoelectric effects in $2005\left[{ }^{2}\right], \mathrm{BiFeO}_{3}$ has become the most widely investigated single-phase ferroelectric magnet owing to the simultaneous presence of ferroelectric and magnetic order at and above room temperature $\left[{ }^{3}\right]$. The ability to tune the electric properties by an applied magnetic field or the possibility to manipulate the magnetic state by an electric field provides an extra functionality that can be exploited for the construction of novel multifunctional devices based on mutual control of magnetic and electric states. $\left[{ }^{4,5}\right]$

Although the first principle calculations $\left[^{6}\right]$ and some experimental works $\left[{ }^{7,8,9}\right]$ suggested that the multiferroic properties of $\mathrm{BiFeO}_{3}$ would be promising for such applications, there are several problems that limit the commercial viability of $\mathrm{BiFeO}_{3}$-based devices, including the low electrical resistivity $\left[{ }^{10,11}\right]$ and the weak magnetoelectric coupling $\left.{ }^{[1,12}\right]$. To overcome the aforementioned problems in $\mathrm{BiFeO}_{3}$, a variety of advanced processing strategies and innovative chemical approaches has been proposed in the literature. Among them, a partial substitution of rare-earth (RE) ions for bismuth is reported as one of the most effective ways to reduce leakage currents and suppress the cycloid-type spatial spin modulation at the same time. $\left[{ }^{13,14,15,16}\right]$ In our earlier work $\left[{ }^{17}\right]$, we showed that the A-site substitution by dysprosium is able to eliminate the formation of secondary phases, improve the dielectric properties and induce a weak ferromagnetism at room temperature. Troyanchuk et al. $\left[{ }^{18}\right]$ have reported that the appearance of the weak ferromagnetic moment in rare-earth doped $\mathrm{BiFeO}_{3}$ ceramics is closely related to the presence of structural phases with other symmetry types than that in pure $\mathrm{BiFeO}_{3}$. These phases are suggested to restore the collinear antiferromagnetic order, in which a weak ferromagnetism may occur due to a conventional interaction of the Dzyaloshinskii-Moryia (DM) type responsible for the weak ferromagnetism of orthoferrites. $\left[{ }^{19}\right]$ The magnetic transition towards commensurate order driven by a structural transformation was found to be dependent on the ionic radius and concentration of the substituting RE element. $\left[{ }^{20}\right]$ The size of RE ions controls the stability of the structural phase; the phenomenon is known in literature as "the internal chemical pressure".[4,21] The smaller the rare-earth ion or the larger the difference 
between the ionic radii of $\mathrm{RE}^{3+}$ and $\mathrm{Bi}^{3+}$ the stronger the chemical pressure and the lower the concentration range of the phase stability for the rhombohedral phase in rare-earth substituted $\mathrm{BiFeO}_{3}$. In this context, $\mathrm{Tb}$ substitution appears to be an interesting case as (i) the large difference in ionic radii of $\mathrm{Bi}^{3+}\left(\mathrm{r}=1.36 \AA\right.$ in 12-fold coordination, $\left.\mathrm{CN}=12\left[{ }^{22}\right]\right)$ and $\mathrm{Tb}^{3+}(\mathrm{r}=$ $1.25 \AA, \mathrm{CN}=12\left[{ }^{23}\right]$ ) may introduce a significant structural distortion via the internal chemical pressure effect, and (ii) a large own magnetic moment carried by $\mathrm{Tb}^{3+}$ ions can give rise to strong magnetic interactions, as previously observed in $\mathrm{TbFeO}_{3}\left[{ }^{24}\right]$.

Surprisingly, despite the considerable interest devoted to the other rare earth metals in recent years, only a limited number of studies exist in the literature for $\mathrm{Tb}$-substituted $\mathrm{BiFeO}_{3}$. Troyanchuk et al. $\left[{ }^{18}\right]$ have prepared a series of $\mathrm{Bi}_{1-\mathrm{x}} \mathrm{Tb}_{\mathrm{x}} \mathrm{FeO}_{3}$ ceramics; they found that upon doping, the parental $R 3 c$ phase first transformed partly into an antipolar Pnam phase at $\mathrm{x}>0.08$; the maximum amount of the orthorhombic Pnam phase is present in the $\mathrm{x}=0.115$ sample; and then, at $\mathrm{x}=0.18$, the biphasic $(R 3 c+$ Pnam $)$ structure evolved into the non-polar orthorhombic Pnma symmetry. The same limiting concentration of $\mathrm{x}=0.08$ was reported by Jiang et al. $\left.{ }^{25}\right]$ for the stability of the polar $R 3 c$ phase in the hot-pressed $\mathrm{Bi}_{0.95-\mathrm{x}} \mathrm{La}_{0.05} \mathrm{~Tb}_{\mathrm{x}} \mathrm{FeO}_{3}$ ceramics. However, in their work, a new phase was found to coexist with the rhombohedral $R 3 c$ phase in a narrow concentration range $0.08 \leq \mathrm{x} \leq 0.10$ and it was suggested to be of a tetragonal symmetry. Additional contradictory and scattered data on structural state and physical properties of terbium-substituted $\mathrm{BiFeO}_{3}$ were noticed in other previous studies $[26,27,28,29,30,31,32]$, most likely because of different processing conditions. This situation has motivated us to prepare the $\mathrm{Tb}$-substituted $\mathrm{BiFeO}_{3}$ ceramics by the advanced processing technique of spark plasma sintering (SPS), which has been recently proven to be an efficient technology to fabricate high-density pure $\mathrm{BiFeO}_{3}$ and $\mathrm{BiFeO}_{3}$-related ceramics, with improved phase purity and enhanced dielectric and multiferroic properties $\left[{ }^{33}\right]$.

In this work, we systematically investigate the effect of terbium A-site substitution on evolution of the crystal and electronic structures, and on the characteristics of the electrical and magnetic behavior in the SPS processed $\mathrm{Bi}_{1-\mathrm{x}} \mathrm{Tb}_{\mathrm{x}} \mathrm{FeO}_{3}(0 \leq \mathrm{x} \leq 0.30)$ ceramic series. The results of the XRD, Raman spectroscopy, ELNES and NEXAFS spectroscopy, differential scanning calorimetry, and magnetic measurements suggest that the appearance and enhancement of the magnetization can be correlated with the composition-driven phase transition of the polar rhombohedral phase to the non-polar orthorhombic phase, which takes place over the concentration range $0.10 \leq \mathrm{x} \leq 0.20$ via the formation of an intermediate state. 


\section{Experimental methods}

\section{Material synthesis}

The polycrystalline samples of the $\mathrm{Bi}_{1-\mathrm{x}} \mathrm{Tb}_{\mathrm{x}} \mathrm{FeO}_{3}(0 \leq \mathrm{x} \leq 0.30)$ series were synthesized by the mixed-oxide route using high-purity oxides of $\mathrm{Bi}_{2} \mathrm{O}_{3}, \mathrm{Fe}_{2} \mathrm{O}_{3}$ and $\mathrm{Tb}_{2} \mathrm{O}_{3}$ (Sigma-Aldrich, $\geq$ 99.99\%). The dried ( $420 \mathrm{~K}$ for $3 \mathrm{~h}$ ) oxides were weighed in stoichiometric proportions and thoroughly mixed for $2 \mathrm{~h}$, using a planetary ball mill (Fritsch, model Pulverisette 7, Germany), in polyamide mortar filled with the $\mathrm{ZrO}_{2}$ milling balls and isopropyl alcohol as a liquid medium. After drying the slurry, the powder mixture was calcined in an alumina crucible at $923 \mathrm{~K}$ for 2 $\mathrm{h}$ in air. An additional homogenization and refining of the precursors was carried out by repeating the process of ball-milling and calcination. The finely ground calcined powders were sintered in air in closed alumina crucible at different temperatures $(1080-1155 \mathrm{~K})$ for $2-6 \mathrm{~h}$. To produce dense ceramics the sintered samples were ground into a very fine powder and then placed in a cylindrical graphite die with a $20 \mathrm{~mm}$ inner diameter. The powder and the die wall were separated by a carbon foil. The consolidation was carried out in vacuum for $3 \mathrm{~min}$ at a maximum temperature of 1023 - $1085 \mathrm{~K}$ and uniaxial stress of $50 \mathrm{MPa}$ using an SPS furnace (FCT Systeme, model HPD 25/1, Germany). The heating rate was set to $200 \mathrm{~K} / \mathrm{min}$. After the SPS consolidation, the power and pressure were switched off and the samples were allowed to cool in the SPS chamber to room temperature naturally. The SPS processed (denoted SPS'ed hereafter) pellets were subjected to an additional heat treatment in air at $900 \mathrm{~K} / 10 \mathrm{~h}$ in order to remove carbon that could infiltrate into the samples during SPS processing at high temperatures.

\section{X-ray diffraction}

Phase analyses and characterization of the crystal structure of the SPS'ed ceramics were performed at room temperature by X-ray diffraction (XRD, PANalytical, model Philips X'Pert Pro, Netherlands) with $\mathrm{Cu} \mathrm{K} \alpha$ radiation. Scans were collected over an angular range $20^{\circ} \leq 2 \theta \leq$ $120^{\circ}$ in continuous scan mode with a step of $0.0167^{\circ}$ and $20 \mathrm{~s}$ collection time per step. The obtained diffraction data were further analyzed by the Rietveld refinement method using a FullProf software package $\left[{ }^{34}\right]$.

\section{Raman spectroscopy}


The room-temperature Raman scattering spectra were collected from an unpolished surface of the SPS'ed pellets using a LabRam micro-probe system (ISA/Jobin-Yvon/Horiba, France) with an excitation source of Ar ion laser line (wave-length $488 \mathrm{~nm}$ ) in back-scattering geometry. Focusing of the laser beam and collection of the scattered light was done by a 100x microscope objective lens. The power of laser spot (a diameter of about $1 \mu \mathrm{m}$ ) on the pellet surface was kept below $3 \mathrm{~mW}$ to prevent a local overheating. The data were collected by a Peltier-cooled charged coupled device camera within a spectral range of $50-700 \mathrm{~cm}^{-1}$ with a step of $0.5 \mathrm{~cm}^{-1}$ and an integration time of $10 \mathrm{~s}$.

\section{Scanning electron microscopy}

For microstructure observations, the SPS'ed samples were mirror-polished and thermally etched at $1040 \mathrm{~K}$ for $20 \mathrm{~min}$. A scanning electron microscope (SEM, a cold field-emission microscope JEOL, model JSM 7000F, Japan) upgraded with an energy dispersive X-ray spectrometer (Oxford Instruments, UK) was used to examine morphology, grain size and a local element occupancy. An apparent density of the sintered ceramics, as measured by Archimedes's method, was about $96 \%$ of the theoretical density. Such a value is higher than that ( 92-94\%) reported for $\mathrm{BiFeO}_{3}$-based ceramics obtained by conventional sintering [ $\left.{ }^{17,35}\right]$.

\section{High-resolution transmission electron microscopy and ELNES spectroscopy}

Cross-sectional samples for transmission electron microscopy (TEM) were prepared using a conventional polishing method. After dimple grinding and polishing, the final thinning to the electron transparency was achieved by low energy low angle ion beam milling. Atomic resolution high angle annular dark field (HAADF) images and ELNES data were taken from the same areas of the sample using the aberration corrected FEI Titan Themis 300. The microscope was operated in scanning TEM (STEM) mode at an accelerating voltage of $200 \mathrm{kV}$ and a probe current of $0.12 \mathrm{nA}$. The Gatan Enfinium 977ER spectrometer was used for acquisition of the EEL spectra. The beam convergence semi-angle was set at $22 \mathrm{mrad}$ and the EEL spectrometer collection semi-angle at $10.3 \mathrm{mrad}(2.5 \mathrm{~mm}$ aperture $)$. Semi-angular collection range of the HAADF detector and annular bright field (ABF) detector was $129-200$ mrad and $15-28 \mathrm{mrad}$, respectively. $\left[{ }^{36,37}\right]$ The bright field (BF) detector collected data within a semi-angle of $13 \mathrm{mrad}$ and/or $73 \mathrm{mrad}$.

\section{XANES spectroscopy and model calculations}


X-ray absorption fine structure (XAFS) spectroscopy measurements were carried out at the ID26 undulator beamline $\left[{ }^{38}\right]$ at the electron storage ring ESRF (Grenoble, France) operating in sixteen bunch mode, with an approximated photon flux of 1011 photons/s with a bandwidth of $0.1 \%$. The incident beam energy was monochromatized with a $\mathrm{Si}<111>$ double crystal monochromator. For all spectra, a metallic Fe reference foil was used to provide an energy calibration for the monochromator. Silicon mirror was used for the rejection of the harmonics coming from the incident X-ray beam. The samples were positioned at $45^{0}$ with respect to the incident beam of cross-section $0.2 \times 0.7 \mathrm{~mm}$. An emission spectrometer with five crystal analyzers $\left(5 \times \mathrm{Ge}<440>, \mathrm{R}=1002 \mathrm{~mm}\right.$ for $\mathrm{Fe} \mathrm{K}_{\alpha 1}$ detection) was used to Bragg select a small portion of the characteristic fluorescence line around the maximum intensity (bandwidth around $1 \mathrm{eV}$ ). This detection method results in better resolved spectral features (high energy resolution fluorescence detected X-ray absorption spectroscopy) and optimal suppression of background. The scans were acquired at $25 \mathrm{~K}$ (sample in He cryostat) to reduce thermal motion of the sample (a dynamical part of the Debye-Waller factor). The NEXAFS spectroscopy scan range was 7090-7189 eV at the Fe K-edge and for the extended XAFS (EXAFS) experiments 6960-7934 $\mathrm{eV}$. The measured X-ray absorption cross-sections were analyzed using the FEFF8.1 (a program for ab-initio real-time multiple scattering (RSMS) calculations of the X-ray absorption fine structure) $\left[{ }^{39}\right]$ and Viper (a program for XAFS data processing and refinement) codes $\left[{ }^{40}\right]$.

\section{Thermal analysis and magnetic measurements}

The field- and temperature-dependence of magnetization was traced using a Magnetic Properties Measurement System (Quantum Design, model MPMS-XL-5, USA) equipped with a superconducting quantum interference device (SQUID) magnetometer and superconducting magnet $( \pm 5 \mathrm{~T})$. The magnetization vs. applied magnetic field curves were measured at three different temperatures 300,150 and $5 \mathrm{~K}$. The dependence of magnetization on temperature was measured over a wide temperature range (5-300 K) under field cooling (FC) / field heating (FH) and zero-field cooling (ZFC) / FC conditions. The applied magnetic field was 0.01, 0.2 and 1 T. Differential scanning calorimetry (DSC) was employed to determine the antiferromagnetic Néel $\left(T_{N}\right)$ and ferroelectric Curie $\left(T_{c}\right)$ temperatures of the SPS'ed ceramics. The changes in heat flow were analyzed using a high-temperature calorimeter (NETZSCH, model Jupiter STA 449$\mathrm{F} 1$, Germany) in the temperature range of $300-1120 \mathrm{~K}$ in air at a controlled heating/cooling rate of $10 \mathrm{~K} / \mathrm{min}$. 


\section{Results and discussion}

\section{X-ray diffraction analysis}

Fig. 1 shows the room-temperature XRD data obtained for the SPS'ed ceramic samples of the $\mathrm{Bi}_{1-\mathrm{x}} \mathrm{Tb}_{\mathrm{x}} \mathrm{FeO}_{3}$ solid solution system for $\mathrm{Tb}$ concentrations within the range $0 \leq \mathrm{x} \leq 0.30$. The peak intensity ratios in the presented diffractograms are indicative of ceramics with a good crystallinity. At first sight, by comparing the stacked XRD patterns in Fig. 1a, one can spot an apparent change in diffraction profiles for different $\mathrm{Tb}$ contents, involving a shift of peak positions, an increase in the peaks' width and the disappearance of some parental peaks along with the appearance of new peaks on progressive Tb substitution. The shifting of peaks towards higher $2 \theta$ angles (see the enlarged views of the XRD patterns for selected $2 \theta$ ranges, Figs. $1 \mathrm{~b}-$ 1c) with increasing $\mathrm{Tb}$ doping content indicates a reduction in $d$-spacing which directly relates to the unit cell shrinkage. The broadening of peaks may possibly originate from a decrease in grain size, which is promoted by Tb addition. SEM micrographs in the insets of Fig. 2 clearly demonstrate the inhibiting effect of Tb substitution on grain growth in the microstructure of the ceramics. Muneeswaran et al. $\left[{ }^{41}\right]$ have shown using the XRD and TEM methods that the crystallite size decreases alongside the particle size with increasing $\mathrm{Tb}$ content in $\mathrm{BiFeO}_{3}$ nanoparticles. An agreement between the size of particles, as determined from TEM, and that of crystallites, as estimated from XRD peak broadening, was obtained for the $\mathrm{Bi}_{1-\mathrm{x}} \mathrm{Tb}_{\mathrm{x}} \mathrm{FeO}$ ceramics series. $\left[{ }^{42}\right]$ Our calculations, using the Debye-Scherrer formula on the measured XRD data, confirm that the average crystallite size decreases upon terbium substitution. The estimated size of crystallites of the pure $\mathrm{BiFeO}_{3}$ ceramics was found to be $70-80 \mathrm{~nm}$, while the broadening of the XRD peaks of the $\mathrm{x} \geq 0.20$ samples indicates that highly doped ceramics comprise of 40-50 nm crystallites. Similar trends in decreasing both the grain size and crystallite size upon the A- site rare earth substitution were reported for Dy-doped $\mathrm{BiFeO}_{3}$ ceramics.[ $[3,44$ ] Besides the observed right shift in $2 \theta$ positions and peaks broadening, the reflections associated with pure $\mathrm{BiFeO}_{3}$ phase show a continuous decrease in intensity as the concentration level of Tb increases. Ultimately, for the $\mathrm{x}=0.30$ ceramic, the two most intense peaks around $2 \theta \approx 32^{0}$

(Fig. 1c), as well as the prominent peak at $2 \theta \approx 38^{0}$ of pure $\mathrm{BiFeO}_{3}$ disappear completely. On the other hand, there is a distinct peak at $2 \theta \approx 25^{\circ}$ in the XRD patterns of the samples with $\mathrm{x} \geq$ 0.15 which cannot be observed in pure $\mathrm{BiFeO}_{3}$. Such a development in diffraction characteristics indicates a substantial structural modification in the SPS'ed Tb-doped $\mathrm{BiFeO}_{3}$ ceramics. 
Phase analysis of pure $\mathrm{BiFeO}_{3}$ ceramics (Fig. 1a) showed that the major perovskite phase displays a pattern characteristic of a rhombohedrally distorted perovskite structure $(R 3 c$ space group). The signature diffraction peaks match well with the standard crystallographic data reported for the rhombohedral $\mathrm{BiFeO}_{3}$ in the Crystallography Open Database $\left[{ }^{45}\right]$ under the COD file No. 4336776. Along with the main $R 3 c$ perovskite phase, traces of a minor impurity phase of the $\mathrm{Bi}_{2} \mathrm{Fe}_{4} \mathrm{O}_{9}$-like structure (COD file \#9008148) are detected as extra, low-intensity diffraction peaks around $2 \theta \approx 28^{0}$ and $33^{\circ}$ in the XRD pattern. The $\mathrm{Bi}_{25} \mathrm{FeO}_{39}$ sillenite and $\mathrm{Bi}_{2} \mathrm{Fe}_{4} \mathrm{O}_{9}$ mullite phases have been reported in literature as typical processing by-products of the solid-state synthesis of $\mathrm{BiFeO}_{3}$ owing to a peculiar kinetics of phase formation in the $\mathrm{Bi}_{2} \mathrm{O}_{3}$ - $\mathrm{Fe}_{2} \mathrm{O}_{3}$ system. $\left.{ }^{46,47}\right]$ Upon $\mathrm{Tb}$ substitution, the small-intensity Bragg reflections of the secondary phase disappear, which implies that a partial replacement of $\mathrm{Bi}^{3+}$ ions for $\mathrm{Tb}^{3+}$ ions stabilizes the formation of the perovskite phase during the conventional solid state reaction, and so it substantially reduces the amount of impurities in final polycrystalline product. Similar observations on substitution-enhanced phase purity were reported for La- $\left[{ }^{48}\right]$, Dy- $\left[{ }^{17}\right]$ and Gd$\left[{ }^{49}\right]$ doped $\mathrm{BiFeO}_{3}$ ceramics. The diffraction profile of highly doped samples $(x \geq 0.20)$ was found to coincide closely with that of orthorhombic $\mathrm{TbFeO}_{3}$ (COD file \#1008090). A weak signature of the emerging Pnma-type structure can be discerned for $\mathrm{Bi}_{0.9} \mathrm{~Tb}_{0.1} \mathrm{FeO}_{3}$, in which double peaks (101)/(020) and (202)/(040) as well as a new (111) orthorhombic peak appear along with the dominant rhombohedral phase peaks in the XRD pattern. The substitutioninduced suppression of the parental $R 3 c$ phase and creation of a new Pnma structure in the $\mathrm{Bi}_{1}$ ${ }_{x} \mathrm{~Tb}_{\mathrm{x}} \mathrm{FeO}_{3}$ system clearly demonstrate the ion size effect $\left[{ }^{21,50}\right]$. An introduction of small $\mathrm{Tb}^{3+}$ ions into $\mathrm{BiFeO}_{3}$ perovskite is expected to reduce the tolerance factor since the average A-site ionic radius is decreased. To relieve the substitution-induced lattice stress, an essential change of the lattice parameters and unit-cell proportions must occur on $\mathrm{Tb}$ doping, leading to the observed structural transformation in the $\mathrm{Bi}_{1-\mathrm{x}} \mathrm{Tb}_{\mathrm{x}} \mathrm{FeO}_{3}$ system.

To further analyze the composition-driven phase transition in $\mathrm{Bi}_{1-\mathrm{x}} \mathrm{Tb}_{\mathrm{x}} \mathrm{FeO}_{3}$ ceramics, the measured XRD patterns were simulated by the Rietveld refinement method. The reflections of the perovskite phase of pure $\mathrm{BiFeO}_{3}$ ceramics were successfully indexed using the $R 3 c$ structural model in a hexagonal setting (in Supplementary material, Fig. S1a). The refined lattice constants, $a=b \approx 5.5782(1) \AA$ and $c \approx 13.8691(2) \AA$, are in accordance with those reported in literature for the rhombohedral $\mathrm{BiFeO}_{3}\left[{ }^{51,52}\right]$. Similarly, a very good agreement between the measured XRD data and simulated $R 3 c$ diffraction profile was obtained for the $\mathrm{x}$ $=0.05$ sample (not shown here). The refined lattice constants are summarized in Table S1 
(Suppl.). The atomic coordinates, bond lengths, bond angles and reliability $R$-factors are listed separately in Table S2 (Suppl.). By comparing the lattice parameters obtained for pure $\mathrm{BiFeO}_{3}$ and $\mathrm{Bi}_{0.95} \mathrm{~Tb}_{0.05} \mathrm{FeO}_{3}$ ceramics, one can see that the incorporation of a small amount of $\mathrm{Tb}$ ions causes a slight volume shrinkage of the rhombohedral unit cell $(\approx 0.5 \%)$, which is associated with a tiny shortening $(\approx 0.1-0.2 \%)$ of both the $a$ and $c$ cell constants. The Rietveld analysis of diffraction data collected for the compositions with highest contents of terbium $(x=0.25$ and 0.30) revealed a single-phase structure which is isostructural to the orthorhombic Pnma phase with $\sqrt{ } 2 a_{p} \times 2 \sqrt{ } 2 a_{p} \times 4 a_{p} \times$ superlattice ( $a_{p}$ is a parameter of pseudocubic unit cell) characteristic of rare-earth magnetic orthoferrites $\mathrm{REFeO}_{3}\left[{ }^{53}\right]$. The refined XRD pattern along with experimental data for the $\mathrm{Bi}_{0.7} \mathrm{~Tb}_{0.3} \mathrm{FeO}_{3}$ ceramics is shown in Fig. S1b (Suppl.). Since the oxygen sublattice in the nonpolar Pnma structure can be described in terms of the $a^{-} b^{+} a^{-}$ octahedral tilt configuration of Glazer $\left[{ }^{54}\right]$, the A-site terbium substitution is thought to give rise to an additional cooperative rotation of the adjacent oxygen octahedra $\left(\mathrm{FeO}_{6}\right)$ around the pseudocubic [111 $]_{\mathrm{C}}$ axis, so that one anti-phase tilt in the polar $R 3 c$ phase ( $a^{-} a^{-} a^{-}$tilt system in the Glazer's notation) is replaced by an in-phase tilt. Considering that the orthorhombic Pnma phase is favored by tilt distortions of the oxygen octahedra which, at their turn, are promoted by small size $\mathrm{Tb}$ ion occupying the A-site positions, the reduced phase stability for the $R 3 \mathrm{c}$ phase in $\mathrm{Tb}$-doped $\mathrm{BiFeO}_{3}$, if compared with other systems doped by larger rare-earth elements (e.g., La-doped $\mathrm{BiFeO}_{3}$ ), can be explained by the larger space that is available in the perovskite cell for tilting. The lattice parameters derived from the Rietveld refinement of the diffraction data for the $\mathrm{x}=0.25$ and 0.30 ceramics are given in Table S1 (Suppl.). A step-like contraction of the primitive cell volume $(\sim 3 \%)$ at $\mathrm{x}=0.10$ (Fig. 2$)$ indicates that the structural transition from the rhombohedral $R 3 c$ phase to the orthorhombic Pnma phase is a first order one. Therefore, according to the Gibbs phase rule, there should exist an intermediate structural state along the concentration range where both the phases coexist. Indeed, a more complex character of diffraction pattern, suggesting the presence of more than one structural phase, was observed for samples within the concentration range $0.10 \leq x \leq 0.20$. Naturally, following the results of refinements for pure and highly-doped $\mathrm{BiFeO}_{3}$, the XRD data obtained for the compounds with compositions in this intermediate interval were tested for the simultaneous presence of the rhombohedral $R 3 c$ and orthorhombic Pnma phase using a two-phase $(R 3 c+P n m a)$ structural model. Fig. S1c (Suppl.) shows the comparison between the measured and simulated XRD pattern for the representative biphasic $\mathrm{x}=0.15$ sample. As it can be seen, the model allowed all the observed reflections to be satisfactorily reproduced with generally small $R$-values (Table 
S2) and the goodness of the fit $\left(\gamma^{2}\right)$ factor below 1.5, thus evidencing that the measured XRD profile results from a superposition of Bragg reflections from two structural components, the rhombohedral $R 3 c$ phase and orthorhombic Pnma phase. The coexistence of the two structures with a dominant diffraction contribution $(\sim 90 \mathrm{wt} . \%)$ from the rhombohedral phase was observed for the $\mathrm{x}=0.10$ ceramics. A minor Pnma phase with orthorhombic cell parameters $a$ $\approx 5.623 \AA, b \approx 7.817 \AA$ and $c \approx 5.420 \AA$ turns into the dominating one as the Tb content is increased to $x=0.15$. Using the mixed-phase approach, stable refinements with $\sim 49$ wt. $\%$ and $\sim 91$ wt.\% of the orthorhombic Pnma phase were obtained for the $\mathrm{x}=0.15$ and $\mathrm{x}=0.20$ samples, respectively. Structural information obtained from the Rietveld analyses of the biphasic sample is summarized in Table S1 (lattice parameters) and Table S2 (atomic coordinates and other structural parameters).

The results of the XRD study demonstrate that the structure of $\mathrm{Bi}_{1-\mathrm{x}} \mathrm{Tb}_{\mathrm{x}} \mathrm{FeO}_{3}$ evolves from the rhombohedral $R 3 c$ phase to the orthorhombic Pnma phase via an intermediate two-phase state, which exists over a wide concentration range $0.10 \leq x \leq 0.20$. Taking into account that the polar $R 3 c$ phase is mainly responsible for ferroelectricity and that the non-polar Pnma phase exclusively enhances magnetization $\left[{ }^{55,56}\right]$, the interval of the phase coexistence or the so called morphotropic phase boundary (MPB) determines the limits of terbium substitution for tailoring the multiferroic properties of $\mathrm{Tb}$-doped $\mathrm{BiFeO}_{3}$. It is worth mentioning that the MPB region of the SPS'ed $\mathrm{Bi}_{1-\mathrm{x}} \mathrm{Tb}_{\mathrm{x}} \mathrm{FeO}_{3}$ ceramics is wider than that reported for the conventional sintered ceramics (for instance, $x=0.10-0.175$ in Ref. $\left[{ }^{27}\right]$ ). The expansion of the MPB boundaries is likely due to local structural defects produced by inhomogeneous thermal gradients or nonuniform temperature distribution which may appear during the SPS processing of complex oxide ceramics $\left[{ }^{57}\right]$.

\section{Raman scattering analysis}

As a complementary technique to X-ray diffraction, Raman spectroscopy has been employed in our comprehensive study of the composition-driven phase transition behavior and local structure modulations in the $\mathrm{Bi}_{1-\mathrm{x}} \mathrm{Tb}_{\mathrm{x}} \mathrm{FeO}_{3}$ ceramic system. Fig. 3 displays the roomtemperature Raman data collected for the Tb-substituted $\mathrm{BiFeO}_{3}$ ceramics in the spectral range $50-700 \mathrm{~cm}^{-1}$. The measured spectra, analogous to the XRD patterns, undergo noticeable changes upon doping. The first obvious feature is the drop in intensity and the broadening of the Raman peaks below $200 \mathrm{~cm}^{-1}$ with $\mathrm{x}$ increasing from 0 up to 0.20 . At $\mathrm{x}=0.20$, the three most intense peaks of pure $\mathrm{BiFeO}_{3}$, located below $200 \mathrm{~cm}^{-1}$, almost disappear and instead a 
broad peak centered at about $300 \mathrm{~cm}^{-1}$ emerges. For the compositions with $\mathrm{x}>0.20$, the firstorder Raman spectrum is dominated by the $300 \mathrm{~cm}^{-1}$ peak. The second noticeable effect of the $\mathrm{Tb}$ substitution is that the position of the fourth most intense peak of $\mathrm{BiFeO}_{3}$ just above 200 $\mathrm{cm}^{-1}$ shifts drastically towards higher wave numbers.

To correlate these changes with the substitution-induced structural phase transition, the Raman scattering spectra were fitted based on an approach of a sum of individual classical damped (but not heavily) harmonic oscillators contributing to the measured spectral response. On decomposing the fitted Raman lines into individual Gaussian components, the peak position of each component, i.e. the natural frequency of each Raman active phonon mode (in $\mathrm{cm}^{-1}$ ) was obtained. Table S3 provides a list of the signature $R 3 c$ Raman modes for the representative $\mathrm{Tb}$ doped samples along with modes of pure $\mathrm{BiFeO}_{3}$. The peak assignment was performed according to earlier reported phonon modes of vibration spectra of pure $\mathrm{BiFeO}_{3}\left[{ }^{58}\right]$. For the rhombohedral $R 3 c$ ferroelectric phase, factor-group analysis of the lattice vibrations predicts $\Gamma_{\text {Raman }}(R 3 c)=9 E+4 A_{l}=13$ Raman active modes in the zone center $(k \approx 0)$, which are split into longitudinal (LO) and transverse (TO) branches due to the polarity of the $R 3 c$ structure. Hlinka et al. $\left.{ }^{[59}\right]$ have emphasized the relevance of crystal surface orientation in the assignment of individual Raman modes of polycrystalline $\mathrm{BiFeO}_{3}$ due to the angular dispersion of longwavelength polar phonon modes. They showed that the Raman spectrum of $\mathrm{BiFeO}_{3}$ ceramics with random orientation of optically anisotropic grains has only 13 phonon peaks, nine of them correspond to the $9 E(\mathrm{TO})$ frequencies and the four remaining modes are the $A_{l}(\mathrm{LO})$ singlets, when the angle $\phi$ between the incident laser beam and the orientation of the spontaneous ferroelectric polarization is zero. With increasing $\phi$ up to $90^{\circ}$ the $E(\mathrm{TO})$ modes split at a variable intensity into one frequency independent TO branch and one dispersive "oblique mode" LO branch. In addition, the $A_{l}$ modes transform completely into transversal optical modes making in total the 22 polar mode branches, $9 E(\mathrm{TO})+9 E(\mathrm{LO})+4 A_{l}(\mathrm{TO})$, observable in the Raman scattering spectrum for perpendicular configuration of the $\mathrm{BiFeO}_{3}$ crystallite face and incoming laser light direction. Our results for pure $\mathrm{BiFeO}_{3}$ are in qualitative agreement with the peak structure reported in Ref. $\left[{ }^{29}\right]$ for ferroelectric phase spectra of a polycrystalline $\mathrm{BiFeO}_{3}$ measured at $\phi \approx 55^{0}$ (i.e., the surface of the sample is randomly oriented along one of the [100], [010], or [001] pseudocubic directions). A slight shift in some of the peak positions can be attributed to different preparation methods. SPS processing has been shown to affect oxygen stoichiometry of $\mathrm{BiFeO}_{3}$ ceramics. $\left[{ }^{33}\right]$ Therefore, the SPS-induced changes in oxygen bonding 
and local disorder are thought to be responsible for the observed shifting of the vibrational frequencies of the Raman mode involving oxygen.

The first three most intense peaks at 70, 135 and $167 \mathrm{~cm}^{-1}$ of the Raman spectrum of $\mathrm{BiFeO}_{3}$ ceramics are manifestation of activation of the $E(\mathrm{TO} 1), E(\mathrm{TO} 2)$ and $A_{l}(\mathrm{LO} 1)$ optical phonons, respectively. These low-frequency Raman modes are closely associated with the A-site vibrations $\left[{ }^{58}\right]$ produced in rhombohedral $\mathrm{BiFeO}_{3}$ by the relative motion of $\mathrm{Bi}^{3+}$ ions against the slightly distorted oxygen $\mathrm{FeO}_{6}$ octahedra, i.e. they are caused by oscillations of the $\mathrm{Bi}-\mathrm{O}$ covalent bonds. Ab-initio electronic structure calculations have shown that ferroelectricity in $\mathrm{BiFeO}_{3}$ results from the cooperative displacements of $\mathrm{Bi}^{3+}, \mathrm{Fe}^{3+}$ and $\mathrm{O}^{2-}$ ions from their centrosymmetric positions. $\left.{ }^{[}{ }^{6}\right]$ The largest displacements, attributed mostly to the Bi-O hybridization, are those of the $\mathrm{Bi}^{3+}$ relative to $\mathrm{O}^{2-}$, where a lone pair of $6 s$ electrons in a trivalent $\mathrm{Bi}$ ions forms a space-filling localized lobe and pushes Bi ion away from its neighboring oxygen atom. Hence, the observation of the sharp $E+A_{l}(\mathrm{LO} 1)$ Raman modes suggests the existence of ferroelectric long-range ordering in pure $\mathrm{BiFeO}_{3}$ ceramics.

Upon terbium substitution, the general shape of the Raman spectra with 11 ( $4 A_{l}$ and $7 E$ ) Raman active modes is preserved in the range of $50-700 \mathrm{~cm}^{-1}$ for all the compositions with $\mathrm{x}$ up to 0.20 (Fig. 3). On close inspection, one can find that with increasing $\mathrm{Tb}$ content the scattering intensity of the low-frequency characteristic modes gradually decreases along with significant broadening of the Raman peaks, indicating continuous weakening (damping) of antiphase vibrations between the $\mathrm{A}$-site and $\mathrm{FeO}_{6}$ sublattices due to dilution of $\mathrm{Bi} 6 s^{2}$ lone pairs by the $\mathrm{A}$ site terbium substitution. As a consequence, the correlation length of the polar order is here lower than that in pure $\mathrm{BiFeO}_{3}$. Table $\mathrm{S} 3$ shows that the low frequency $E+A_{l}(\mathrm{LO} 1)$ Raman modes of the $x \leq 0.20$ samples slightly, but continuously shift towards higher frequency values with the increasing $\mathrm{Tb}$ concentration. This tiny blue-shift can be explained by lower atomic mass of $\mathrm{Tb}$, if compared to that of $\mathrm{Bi}^{3+}$ ions. $\left[{ }^{60}\right]$ A much stronger composition dependence can be observed in Fig. 3 for the $A_{l}(\mathrm{LO} 2)$ mode at around $220 \mathrm{~cm}^{-1}$. This longitudinal longfrequency phonon mode is clearly detectable in all our samples with $R 3 c$ phase (pure and biphasic compositions) and can be associated with the octahedral tilt distortion caused by oxygen vibrations $\left[{ }^{58}\right]$. With increasing the amount of $\mathrm{Tb}$, the $A_{l}[111]$ tilt mode shifts from $215 \mathrm{~cm}^{-1}$ in pure $\mathrm{BiFeO}_{3}$ to $\sim 233 \mathrm{~cm}^{-1}$ in the $\mathrm{x}=0.20$ sample. Furthermore, a ratio of the spectral intensities of the $A_{l}(\mathrm{LO} 1)$ and $A_{l}(\mathrm{LO} 2)$ modes was found to decrease (not shown here), which indicates an enhancement in tilting distortion of $\mathrm{FeO}_{6}$ octahedra on $\mathrm{Tb}$ doping. The observed evolution in the Raman mode correlates nicely with structural changes identified by $\mathrm{X}$-ray diffraction, where the addition of $\mathrm{Tb}$ ions was found to enhance the antiphase rotation of 
the oxygen octahedra in the rhombohedral $R 3 c$ phase. From Table S2, one can see that the Fe$\mathrm{O}-\mathrm{Fe}$ bond angle, which is directly related to the rotation angle of the oxygen octahedra, increases as the concentration of $\mathrm{Tb}$ increases up to 20 at. $\%$.

Another feature recognized in the Raman spectra of the doped samples is the appearance of new phonon modes at about $620 \mathrm{~cm}^{-1}$ (see inset of Fig. 3) and $320 \mathrm{~cm}^{-1}$ (for $\mathrm{x} \geq 0.20$ ), which evolve on doping. Bielecki et al. $\left.{ }^{[29}\right]$ have pointed out that the occurrence of the $\Gamma$-point $620 \mathrm{~cm}^{-1}$ phonon is the most sensitive indication of the presence of an orthorhombic phase when isovalent substitutions are carried out on the $\mathrm{A}$ - or $\mathrm{B}$-sites of $\mathrm{BiFeO}_{3}$ perovskites. The observation of this vibration mode in our $\mathrm{Bi}_{1-\mathrm{x}} \mathrm{Tb}_{\mathrm{x}} \mathrm{FeO}_{3}$ system suggests that the orthorhombic Pnma structure begins to emerge along with the rhombohedral phase when $\mathrm{x}$ is close to 0.10 . A coexistence of the two phases on a micron scale in samples within the concentration range $0.10 \leq \mathrm{x} \leq 0.20$ is evidenced in Fig. 3 by the mixed structure of the $R 3 c$ and Pnma phonon modes in the measured Raman spectra. The structural transformation is completed at $\mathrm{x} \approx 0.25$, as the Raman spectrum of the sample is dominated by the broad $320 \mathrm{~cm}^{-1}$ band without any spectral trace of the $R 3 \mathrm{c}$ phase. In accordance with studies on structurally related compounds, the $320 \mathrm{~cm}^{-1}$ Raman band originates from the Pnma A-site vibrations that overlap with the oxygen tilt modes. $\left[{ }^{61}\right]$ As the content of $\mathrm{Tb}$ is further increased to 0.30 , the $320 \mathrm{~cm}^{-1}$ band slightly shifts to lower wave numbers. Such a relatively small red-shift is likely caused by the small orthorhombic unit cell distortion (from XRD refinements, Table $\mathrm{S} 1$ ), reflecting the decrease in $\mathrm{Bi} / \mathrm{Tb}-\mathrm{O}$ bond strength as $\mathrm{Bi}^{3+}$ ions are replaced by smaller $\mathrm{Tb}^{3+}$ ions. It should be mentioned that even the spectrum of the $\mathrm{Bi}_{0.8} \mathrm{~Tb}_{0.2} \mathrm{FeO}_{3}$ sample resembles that of the nonpolar orthorhombic $\mathrm{x}=0.25$ and 0.30 samples, there are small spectral peaks in the low frequency range corresponding to the $E+A_{1}$ Raman modes which, consistently with the results of the XRD analysis, confirm the persistence of weak ferroelectric long range order in this biphasic structure. It also proves that the substitution-induced polar-to-nonpolar transition in the spark plasma processed $\mathrm{Bi}_{1-\mathrm{x}} \mathrm{Tb}_{\mathrm{x}} \mathrm{FeO}_{3}$ ceramics takes place over a wide concentration range $0.10 \leq \mathrm{x} \leq 0.20$.

\section{Thermal DSC analysis - long-range ordering temperatures}

Following the results of structural analyses and taking into account the critical behavior of the order parameters (spontaneous polarization and magnetization), SPS'ed samples of $\mathrm{Bi}_{1}$ ${ }_{x} \mathrm{~Tb}_{\mathrm{x}} \mathrm{FeO}_{3}$ were investigated by differential scanning calorimetry for possible changes in magnetic and ferroelectric phase transitions temperatures that might occur due to a partial replacement of $\mathrm{Bi}^{3+}$ ions for smaller, less-polarizable and magnetic $\mathrm{Tb}^{3+}$ ions. 
The DSC data collected for the pure and $\mathrm{Tb}$-doped $\mathrm{BiFeO}_{3}$ samples in the temperature interval $573-1120 \mathrm{~K}$ in heating and cooling regimes are shown in Fig. 4. As it can be seen in Fig. 4a, $\mathrm{BiFeO}_{3}$ exhibits two distinct thermal effects over the investigated range. On heating, the first endothermic peak occurs at $644 \mathrm{~K}$, which corresponds to the reported antiferromagnetic Néel temperature of rhombohedral $\mathrm{BiFeO}_{3}, \mathrm{~T}_{\mathrm{N}} \approx 645 \mathrm{~K}\left[{ }^{62}\right]$. The $\mathrm{T}_{\mathrm{N}}$ peak (see inset of Fig. 4a) shows an apparent $\lambda$-type curve, indicating a second order transition from an antiferromagnetic phase to a paramagnetic one. Although a small energy change is associated with the magnetic transition, the change of magnetic structure can be identified in the heat flow vs. temperature plot.* The second, much stronger, endothermic effect with onset at around $1100 \mathrm{~K}$ can be related to a transition from the non-centrosymmetric ferroelectric phase to the centrosymmetric paraelectric phase. The sharp peak, centred at $1104 \mathrm{~K}$, compares well with the literature value of the Curie transition temperature of pure $\mathrm{BiFeO}_{3}, \mathrm{~T}_{\mathrm{c}} \approx 1103 \mathrm{~K}\left[{ }^{62}\right]$. In cooling regime, the high temperature peak shifts by $\sim 10 \mathrm{~K}$ towards lower temperatures, while the temperature of the onset of the ferroelectric phase transition and the Néel temperature remain unchanged. The distinct thermal hysteresis in $\mathrm{T}_{\mathrm{c}}$ points to a first order phase transition that arises from an abrupt change of the order parameter due to a structural transformation accompanying the ferroelectric to paraelectric phase transition.

Comparing the DSC curves of the $\mathrm{x}=0$ and 0.05 samples (Suppl., Fig. S2), one can notice a significant drop and broadening in DSC output voltage signal at the Curie temperature when the small amount of the $\mathrm{Bi}^{3+}$ ions is replaced by $\mathrm{Tb}^{3+}$ ions. This effect is observed in both heating (Fig. S2a) and cooling (Fig. S2b) regimes and reflects a suppression of ferroelectric long-range order upon substitution. Another feature observable in thermal behavior of the $\mathrm{x}=0.05$ sample is that the onset temperature of the long-range ferroelectric ordering is lowered to $1088 \mathrm{~K}$ and the thermal hysteresis of $\mathrm{T}_{\mathrm{c}}$ is increased by $\sim 15 \mathrm{~K}$ to $\sim 25 \mathrm{~K}$, if compared with pure $\mathrm{BiFeO}_{3}$. Unlike the ferroelectric Curie temperature, the antiferromagnetic Néel transition temperature of $\mathrm{Bi}_{0.95} \mathrm{~Tb}_{0.05} \mathrm{FeO}_{3}$ ceramics corresponds to that of $\mathrm{BiFeO}_{3}$ ceramics and does not show any significant change in heating/cooling process.

A notable development in DSC curves is observed for samples with $\mathrm{Tb}$ concentrations $\mathrm{x} \geq 0.10$. For the 0.10 sample, one can clearly see (Fig. $4 b$ ) that another endothermic peak $\left(\mathrm{T}_{\mathrm{N}}\right)$ occurs at around $637 \mathrm{~K}$ on heating. Upon further doping, the $\mathrm{T}_{\mathrm{N}}$ ' peak becomes more obvious, while the $\mathrm{T}_{\mathrm{N}}$ peak at $644 \mathrm{~K}$ disappears. Similar trends were observed for exothermic DSC peaks when

\footnotetext{
* Note: It is difficult to absolutely define the actual point of the phase transition in the DSC curve. Different protocols have been adopted in the literature using maximum peak or the onset of the peak at either low scan rates or extrapolated rates. In this work we take the temperature of peak maximum as the transition temperature.
} 
samples are cooled from $1120 \mathrm{~K}$ down to room temperature (Fig. $4 \mathrm{c}$ ). In addition, the new $\mathrm{T}_{\mathrm{N}}$, peak, as contrasted to the $\mathrm{T}_{\mathrm{N}}$ peak, shows a small thermal hysteresis of about $5 \mathrm{~K}$ in heating/cooling run for all the $\mathrm{x} \geq 0.10$ ceramic samples. The occurrence of the $\mathrm{T}_{\mathrm{N}}$, peak in thermal response of $\mathrm{Bi}_{0.9} \mathrm{~Tb}_{0.1} \mathrm{FeO}_{3}$ can be linked with a transition of the canted antiferromagnetic spin structure of the Pnma phase into a paramagnetic spin state, and vice versa (will be discussed in the following section). Very recently, Walker et al. $\left[{ }^{63}\right]$ have reported on the antiferromagnetic-to-paramagnetic transition in $\mathrm{Dy}-\mathrm{Sm}$, and $\mathrm{Gd}$-doped $\mathrm{BiFeO}_{3}$ ceramics, which occurs at the temperature almost identical to that observed in our $\mathrm{Bi}_{1-\mathrm{x}} \mathrm{Tb}_{\mathrm{x}} \mathrm{FeO}_{3}$ ceramic series $\left(\mathrm{T}_{\mathrm{N}}\right)^{\prime}$.

Different thermal features are displayed by the doped samples at the ferroelectric phase transition (Figs. $4 \mathrm{~b}$ and $4 \mathrm{c}$ ). With increasing $\mathrm{x}$ up to 0.20 the $\mathrm{T}_{\mathrm{c}}$ peak shifts to lower temperatures along with the reducing DSC signal intensity for both the heating and cooling regimes. At $\mathrm{x}=$ 0.20 , a sign of the ferroelectric phase transition near $1100 \mathrm{~K}$ (on heating) and $1040 \mathrm{~K}$ (on cooling) can be yet identified in thermal response of the sample. A further addition of Tb results in the disappearance of the high temperature peak (within the detection limit of the DSC analyzer). The shift towards low temperatures, reduction and broadening of the $T_{c}$ peak can be attributed to the suppression of ferroelectric long-range order and to the disorder at the A-sites of $\mathrm{BiFeO}_{3}$ due to the replacement of large $\mathrm{Bi}^{3+}$ ions with smaller and less polarizable $\mathrm{Tb}^{3+}$ ions. It is worth mentioning here that for ceramics with $\mathrm{x} \geq 0.10$ the thermal hysteresis of the $\mathrm{T}_{\mathrm{c}}$ peak is much larger than that of magnetic transition. For instance, in the $\mathrm{x}=0.15$ sample the difference between the endothermic and exothermic $\mathrm{T}_{\mathrm{c}}$ peaks $\left(\Delta \mathrm{T}_{\mathrm{c}}\right.$, ) is about $57 \mathrm{~K}$, while the difference $\mathrm{T}_{\mathrm{N}}$, (heating run) $-\mathrm{T}_{\mathrm{N}}$, (cooling run) is only $7 \mathrm{~K}$. The origin of such a large temperature hysteresis of the ferroelectric phase transition can be attributed to the substitutioninduced disorder at A-site and the pinning effect of oxygen vacancies on ferroelectric domain walls movement $\left[{ }^{64}\right]$.

\section{Room-temperature magnetic properties}

Isothermal magnetization vs. magnetic field $(\mathrm{M}-\mathrm{H})$ dependences recorded for the $\mathrm{Bi}_{1-\mathrm{x}} \mathrm{Tb}_{\mathrm{x}} \mathrm{FeO} \mathrm{O}_{3}$ ceramics $(\mathrm{x}=0,0.05,0.15$ and 0.25$)$ at room temperature and $5 \mathrm{~K}$ are shown in Fig. 5a and Fig. $5 b$, respectively. Although, due to paramagnetic contribution from $\mathrm{Tb}^{3+}$ ions no saturation of the magnetization is observed at room temperature even under the maximum applied field of \pm $5 \mathrm{~T}$, it is obvious from Fig. 5a that $\mathrm{Tb}$ doping gives rise to the appearance and evolution of the spontaneous magnetization. While the pure $\mathrm{BiFeO}_{3}$ sample displays, as expected for an antiferromagnet with a collinear incommensurate antiferromagnetic structure, a linear field- 
dependence of magnetization, the $\mathrm{M}-\mathrm{H}$ curves of the doped samples are nonlinear and hysteretic, with progressive enhancement of the remanent magnetization $\left(\mathrm{M}_{\mathrm{r}}\right)$ on doping. The plot of the remanent magnetization as a function of $\mathrm{Tb}$ content is shown in the inset of Fig. 5a. The room-temperature value of $\mathrm{M}_{\mathrm{r}}$ continuously increases as the amount of $\mathrm{Tb}$ ions increases, reaching a plateau with a maximum value of about $0.34 \mathrm{emu} / \mathrm{g}$ at $\mathrm{x}=0.25$ and 0.30 . The coercivity was found to exhibit a slightly different compositional behavior at room temperature (not shown here). With increasing the amount of $\mathrm{Tb}$ ions the coercive field first increased to a maximum strength of about $0.7-0.8 \mathrm{~T}$ for the $\mathrm{x}=0.15$ and 0.20 samples, and then dropped down to $0.5 \mathrm{~T}$ for $\mathrm{Bi}_{0.7} \mathrm{~Tb}_{0.3} \mathrm{FeO}_{3}$ ceramics. Small, but non-zero magnetic moments and large coercive fields of the $\mathrm{Tb}$-substituted $\mathrm{BiFeO}_{3}$ ceramics are characteristic of a weak ferromagnetic material with a canted antiferromagnetic spin order $\left[{ }^{18,19,62}\right]$. It can be inferred that the appearance and enhancement of the remanent magnetization in the system results from the substitution-induced transition of the canted $G$-type antiferromagnetic structure from cycloidal incommensurate spin ordering to commensurate structure, in which the canted ferromagnetic component of the antiferromagnetically ordered spins becomes measurable. Neutron diffraction experiments and high-resolution X-ray diffraction synchrotron studies revealed a change in the periodicity of the incommensurate magnetic order in $\mathrm{BiFeO}_{3}$ when a structural phase transition occurs upon rare-earth doping. $\left[{ }^{65}\right]$ Zaleskii et al. $\left[{ }^{66}\right]$ by measuring the nuclear magnetic resonance spectra of $\mathrm{La}$-doped $\mathrm{BiFeO}_{3}$ provided a direct evidence of the correlation between the suppression of the spatially modulated spin structure and the rhombohedral-toorthorhombic phase transition. They demonstrated that the orthorhombic Pnma phase restores the collinear antiferromagnetic order, in which a weak ferromagnetism occurs due to the Dzyaloshinskii-Moryia (DM) exchange interaction. The dominant role of the DM-type interaction in the enhancement of the room temperature magnetization of the rare-earth substituted $\mathrm{BiFeO}_{3}$ compounds has been demonstrated experimentally by the improved spin canting of about $0.6^{0}\left[{ }^{67}\right]$. The weak ferromagnetism in the $\mathrm{Bi}_{1-\mathrm{x}} \mathrm{Tb}_{\mathrm{x}} \mathrm{FeO}_{3}$ ceramics likely originates from the restored $G$-type commensurate antiferromagnetic structure, in which the $\mathrm{Fe}^{3+}$ ions are antiferromagnetically ordered along the orthorhombic $a$ axis and the canted net ferromagnetic moment develops upon substitution-induced structural distortion in a direction perpendicular to the antiferromagnetic axis (i.e., along the orthorhombic $c$ axis). Our structural measurements show that upon $\mathrm{Tb}$ substitution the parental $R 3 c$ phase of $\mathrm{BiFeO}_{3}$ gradually transforms to the Pnma phase via an intermediate two-phase structural state existing within the concentration range $0.10 \leq \mathrm{x} \leq 0.20$. Thus, the measured magnetization of about $0.12 \mathrm{emu} / \mathrm{g}$ for the $\mathrm{x}=0.10$ sample can be attributed to the presence of $\sim 10 \mathrm{wt} \%$ of the orthorhombic 
Pnma phase in the R3c+Pnma structure (Table S1). As the amount of the Pnma phase in biphasic compositions increases, the remanent magnetization is expected to increase due to the evolution of the DM-type coupling. Indeed, a magnetic moment of about twice the value of the $\mathrm{x}=0.10$ sample was measured for compositions with the dominant Pnma phase $\left(\mathrm{M}_{\mathrm{r}} \sim 0.21\right.$ and $0.26 \mathrm{emu} / \mathrm{g}$ for $\mathrm{x}=0.15$ and 0.20 , respectively). The structural transition to the Pnma phase is completed within the concentration range $0.20<\mathrm{x}<0.25$, which correlates with the maximum of the remanent magnetization of $0.34 \mathrm{emu} / \mathrm{g}$ measured in $\mathrm{Bi}_{0.75} \mathrm{~Tb}_{0.25} \mathrm{FeO}_{3}$ ceramics. Further incorporation of $\mathrm{Tb}$ ions into the Pnma samples does not affect substantially the weak ferromagnetic state at room temperature, as demonstrated by a value of $\mathrm{M}_{\mathrm{r}}$ of $0.34 \mathrm{emu} / \mathrm{g}$ for the $x=0.30$ sample within the measuring sensitivity of the SQUID magnetometer used. By combining the results of magnetic measurements with those of Raman and DSC studies, one can conclude that the ferroelectric-active composition with the highest $\mathrm{M}_{\mathrm{r}}$ at room temperature corresponds to the $\mathrm{Tb}$ concentration of about 20 at.\%.

The microscopic mechanism of the composition-driven magnetic transition and the appearance of the weak ferromagnetism in the $\mathrm{Tb}$-substituted $\mathrm{BiFeO}_{3}$ can be understood in the context of structural evolution in the $\mathrm{FeO}_{6}$ octahedral tilt system. According to results of the Rietveld analysis of the room-temperature XRD data, structural distortions induced by the replacement of $\mathrm{Bi}^{3+}$ ions with smaller $\mathrm{Tb}^{3+}$ ions at the A-sites are accompanied by variations in the local environment such as Fe-O bond lengths and Fe-O-Fe bond angles (Table S1). In addition, Raman spectroscopy on the doped samples revealed a significant blue-shift in the $A_{l}$ [111] tilt mode (Suppl., Table S3), so confirming the substitution-induced modulation of the $\mathrm{FeO}_{6}$ octahedral tilt. The Fe-O-Fe bond angle, which is closely related to the indirect antisymmetric superexchange coupling between two antiferromagnetic layers of the $\mathrm{Fe}^{3+}$ spins $\left[{ }^{68}\right]$, increases from $153.5^{0}(\mathrm{x}=0)$ to $155.2^{0}(\mathrm{x}=0.20)$ on Tb doping. This suggests a substitution-enhanced canting of the antiferromagnetic ordered spins and increased magnetic interactions.

\section{Weak ferromagnetism and electronic structure}

For a better quantitative understanding of the role of the introduced $\mathrm{Tb}^{3+}$ ions in the appearance and enhancement of the net magnetization, we performed detailed XAFS and STEM-ELNES spectroscopic studies. These techniques are extremely useful for probing the local crystal chemistry and determining the electronic structure at the atomic scale due to their high sensitivity to structural distortions, covalence, and with that, ferroic properties. The RGB composite high-resolution HAADF (blue) image interleaved with the annular bright field, ABF, (yellow) image is shown in Fig. 6a in order to illustrate the local arrangement of the $\mathrm{Bi}$ and $\mathrm{Fe}$ 
cations and light oxygen atoms in $\mathrm{BiFeO}_{3}$. Figs. $6 \mathrm{~b}$ and $6 \mathrm{c}$ display $\mathrm{HAADF}$ images overlaid by the [010] projected crystal structures of the respective $R 3 c$ and Pnma unit cells (insets: Bi purple, $\mathrm{Fe}$ - brown, and $\mathrm{O}$ - red circles) for $\mathrm{BiFeO}_{3}$ and $\mathrm{Bi}_{0.7} \mathrm{~Tb}_{0.3} \mathrm{FeO}_{3}$, respectively. Atomic resolution STEM-HAADF images, confirming the chemical nature of the SPS'ed $\mathrm{Bi}_{1-\mathrm{x}} \mathrm{Tb}_{\mathrm{x}} \mathrm{FeO}_{3}$ samples, are shown in Supplementary material (Figs. S3a - d).

\section{Electronic structure analysis using ELNES spectra}

Fig. 7a shows the ELNES spectra acquired at the $\mathrm{O} K$ core loss edge of the $\mathrm{Bi}_{1-\mathrm{x}} \mathrm{Tb}_{\mathrm{x}} \mathrm{FeO}_{3}(\mathrm{x}=$ 0, 0.15 and 0.30) samples. Details on sample measurements are given in Supplementary material (along with the ELNES Fe $\mathrm{L}_{2,3}$ spectra). The $\mathrm{O} \mathrm{K}$ spectra, to a first approximation, results from the electronic transitions from the $\mathrm{O} 1 s$ core level to unoccupied $\mathrm{O} 2 p$ states arising due to the hybridization of the $\mathrm{O} 2 p$ orbital with the unoccupied Fe $3 d$ orbital configurations and also because of a partial covalent bonding with the A-site (Bi, $\mathrm{Tb}$ ) atoms. $\left[{ }^{69}\right]$ Thus, the $\mathrm{O}$ $\mathrm{K}$ edge can also track the nearest bonding to the $\mathrm{Bi}$ cation that has practically inaccessible ELNES edge (Bi-M edge) with the instrument used in this study. From Fig. 7a, one can clearly see that the overall shape and the fine structure of the $\mathrm{O} K$ edge spectral lines change due to the Tb substitution. The ELNES O K spectrum of the $\mathrm{x}=0$ sample with two characteristic $\mathrm{A}$ and $\mathrm{B}$ bands closely matches the earlier reported data for $\left.\mathrm{BiFeO}_{3} \cdot{ }^{70,71}\right]$ According to the density functional theory (DFT) and RSMS calculations $\left[{ }^{69,70}\right]$, the A band is mainly due to the $1 s \rightarrow 2 p$ transitions and the post-edge $\mathrm{B}$ band involves contributions from both the $\mathrm{Fe}$ and $\mathrm{Bi}$ environments featuring a peak broadening due to $\mathrm{O} 2 p$ states hybridized with the Fe $4 s$ and $4 p$ states. A small shoulder (a broad hump-like feature) located at about $537 \mathrm{eV}$ on the high-energy side of the A peak ( $2 \mathrm{eV}$ above the Fermi level), has been attributed to backscattering on $\mathrm{O}^{2-}$ ions, while also having a contribution from covalent bonding to unoccupied $\mathrm{Bi}$ states, $\mathrm{Bi} 5 \mathrm{~d}$ $\left[{ }^{72}\right]$, Bi $s p\left[{ }^{73}\right]$, or Bi $p$ states $\left[{ }^{70}\right]$. Upon Tb substitution, the degree of the Bi-O hybridization is progressively reduced and, ultimately, for $\mathrm{x} \geq 0.25$ the shoulder completely disappears, as demonstrated by the near-edge structure spectrum of the $\mathrm{x}=0.30$ sample. This is in agreement with the Raman scattering and DSC studies and confirms the loss of ferroelectricity due to the dilution of Bi lone pairs by $\mathrm{Tb}$ doping, as $\mathrm{Tb}^{3+}$ does not have an $\mathrm{n}^{2}$ lone pair that according to DFT calculations $\left[{ }^{6,74}\right]$ is responsible for $98 \%$ of the ferroelectricity in $\mathrm{BiFeO}_{3}$. Comparing the near-edge structures of pure and doped $\mathrm{BiFeO}_{3}$, it is clear that some extra spectral features develop upon adding $\mathrm{Tb}$ to the system. A new separate peak (denoted as A2 hereafter) appears at about $538 \mathrm{eV}$ and increases in intensity upon doping. As in pure $\mathrm{BiFeO}_{3}$, the edge-peak (A1) 
is due to the $\mathrm{O} 2 p$ - Fe $3 d$ hybridization, while the $\mathrm{A} 2$ peak most likely originates from bonding with the rare-earth $d$ states $\left[{ }^{70}\right]$. Saetereli et al. $\left[{ }^{70}\right]$ have shown that in $\mathrm{La}$-doped $\mathrm{BiFeO}_{3}$ the $\mathrm{Bi}$ $p$ states, responsible for the shoulder on the $\mathrm{BiFeO}_{3} \mathrm{~A}$ peak, are too low in energy to contribute to the intensity in the energy range where the A2 feature appears. These $\mathrm{Tb} d$ states are also involved in the increased intensity in the area between peaks A2 and B. Another recognizable feature in the $\mathrm{O} K$ edge of the highly doped $\mathrm{x}=0.30$ sample is the decreased intensity ratio of peak A1 to peak B. Again, in analogy with the near-edge absorption studies of Saeterli $\left[{ }^{70}\right]$ and Abbate $\left[{ }^{75}\right]$ for La-doped $\mathrm{BiFeO}_{3}$, this can be attributed to the lower electronegativity of $\mathrm{Tb}$ compared to $\mathrm{Bi}$ (1.2 vs 2.02 on the Pauling scale), giving a reduction in density of the $\mathrm{Fe} d$ states in this part of the spectrum. According to the RSMS calculations $\left[{ }^{75}\right]$, unoccupied Fe $s$ states, responsible for part of the $\mathrm{B}$ peak in $\mathrm{BiFeO}_{3}$, are in the compositions modified by rare earths located in a sharp band lower in energy than the relevant $\mathrm{O} p$ states and are not as involved in $\mathrm{Fe}-\mathrm{O}$ bonding as in pure $\mathrm{BiFeO}_{3}$. The reduced $\mathrm{O} 2 p-\mathrm{Fe} 4 s$ orbital mixing can be linked with the fact that the broad B peak centered at $544.5 \mathrm{eV}$ is observed to sharpen on $\mathrm{Tb}$ doping. The energy positions and shapes of the ELNES spectra of the $\mathrm{O} K$ edge of the investigated compositions are very similar to that of $\alpha-\mathrm{Fe}_{2} \mathrm{O}_{3}\left[{ }^{69,76}\right]$, confirming the $\mathrm{Fe}^{3+}$ oxidation state in the SPS'ed Tb-substituted samples.

Unlike the $\mathrm{O} \mathrm{K}$ edge, no major change is observed in spectra of $\mathrm{Bi}_{1-\mathrm{x}} \mathrm{Tb}_{\mathrm{x}} \mathrm{FeO}_{3}$ ceramics acquired at the Fe $2 p$ edges (Suppl., Fig. S4). The Fe L 2,3 spectral lines are characterized by two sharp peaks, or "white lines", $\mathrm{L}_{3}$ at $\sim 716 \mathrm{eV}$ and $\mathrm{L}_{2}$ at $\sim 729 \mathrm{eV}$, which respectively originate from transitions between the spin-orbit split-core levels $\mathrm{Fe} 2 p^{3 / 2}$ and $\mathrm{Fe} 2 p^{1 / 2}\left(2 p^{6} 3 d^{5}\right.$ configuration) to empty Fe $3 d$ states $\left(2 p^{6} 3 d^{6}\right)$ above the Fermi level.[77,78 $]$ It has been reported that the $\mathrm{Fe} \mathrm{L}_{2,3}$ edge is sensitive to the valence with the onset energy shifting as the oxidation changes from +2 to $\left.+3 .{ }^{79}\right]$ The overall shape and line structure of $\mathrm{Fe} 2 p$ edges in $\mathrm{Tb}$-doped $\mathrm{BiFeO}_{3}$ match well with those of reference hematite, as earlier reported in Refs [ [ $\left.{ }^{80,81}\right]$. Thus, the measured EEL Fe$\mathrm{L}_{2,3}$ edge spectra indicate that iron is in the $3+$ valence state in all the investigated samples. A closer inspection of the spectra revealed that the white lines of the doped samples increase in intensity, if compared to that of pure $\mathrm{BiFeO}_{3}$, and the $\mathrm{L}_{3}$ peak of the biphasic MPB sample ( $\mathrm{x}$ $=0.15$ ) is shifted by $0.7 \mathrm{eV}$ to lower energies. The red shift of Fe L edge at MPB has been observed by A. Y. Borisevich et al. $\left[{ }^{82}\right]$ in similar Sm-substituted $\mathrm{BiFeO}_{3}$ compounds using the NEXAFS technique and this spectral change was ascribed to a weaker $\mathrm{Fe} 3 d-\mathrm{O} 2 p$ hybridization caused by the modulation of Fe spacings at the MPB.

\section{$\underline{\text { Electronic structure analysis using NEXAFS spectra }}$}


In order to further corroborate the reduced degree of the Fe-O hybridization in Tb-doped $\mathrm{BiFeO}_{3}$, we measured the XAFS spectra at the Fe K edge. The synchrotron X-ray absorption at the Fe $\mathrm{K}$ edge corresponds to the electronic transitions from $1 s$ to $4 p$ states and is sensitive to the octahedral environment and local electrostatic interaction. $\left.{ }^{83,84}\right]$ Thus, the Tb substitutioninduced distortion of the chemical bonding environment of $\mathrm{Fe}-\mathrm{O}-\mathrm{Fe}$ in $\mathrm{BiFeO}_{3}$ perovskite is expected to result in different NEXAFS signatures. Fig. 7b displays the Fe K edge XAFS spectra of the representative $\mathrm{x}=0,0.05,0.15$ and 0.25 samples, and the reference powders of $\alpha-\mathrm{Fe}_{2} \mathrm{O}_{3}$ and $\mathrm{Fe}_{3} \mathrm{O}_{4}$. From Fig. 7b, the spectral similarity of the $\mathrm{Bi}_{1-\mathrm{x}} \mathrm{Tb}_{\mathrm{x}} \mathrm{FeO}_{3}$ samples and $\alpha-$ $\mathrm{Fe}_{2} \mathrm{O}_{3}$ in shape and position is obvious. Thus, the oxidation state of iron in $\mathrm{BiFeO}_{3}$ is indeed $3+$ and does not change substantially on Tb doping, as demonstrated by the nearly fixed position of the absorption edge for samples with varying doping concentration. This observation verifies the results of our ELNES measurements at the $\mathrm{O} K$ and Fe L edges. The fine structure of the $\mathrm{BiFeO}_{3}$ and $\mathrm{BiFeO}_{3}$-related compounds near the $\mathrm{Fe} \mathrm{K}$ edge has been resolved theoretically by analysis of the electronic density of states obtained from first principle DFT calculations. [ ${ }^{[5,86,87,88}$ ] A weak pre-edge peak A corresponds to the dipole-forbidden $1 s \rightarrow 3 d$ transition, which is partially allowed because of electric quadrupole coupling and/or Fe $3 d-4 p$ orbital mixing. The main absorption edge feature (B peak) can be assigned to the purely dipoleallowed electronic $1 s \rightarrow 4 p$ transition. The spectral C, D and E peaks result from the multiplescattering contribution of absorbing $\mathrm{Fe}$ in the $\mathrm{FeO}_{6}$ octahedron surrounded by $\mathrm{Bi}$ and $\mathrm{Tb}$.

Splitting of the spectral peaks, which are associated with the electronic transitions to the Fe $3 d$ states, for instance the pre-edge A peak in Fig. 7b, is typically observed in transition metal oxides $\left[{ }^{77}\right]$ and $\mathrm{BiFeO}_{3}$-type perovskites $\left[{ }^{83,89}\right]$ and can be attributed to the so called " $d$-orbital splitting". This phenomenon is referred to as the splitting of the half-filled $\mathrm{Fe}^{3+} d$ states into two energy levels, the three degenerate nonbonding $t_{2 g}$ and the two degenerate anti-bonding $e_{g}$ orbitals, by the strong crystal field (or ligand field) due to its octahedral coordination. The $d$ orbital splitting is triggered by the local Coulomb electrostatic interaction of the $\mathrm{O} s p$ with the Fe $3 d$ orbitals $\left[{ }^{77}\right]$. The energy difference between the $t_{2 g}$ and $e_{g}$ orbitals is called the octahedral crystal field splitting energy $\left(\Delta_{0}\right)$. It is an important spectral factor that determines the spin configuration of the transition metal complex, so its magnetism. The value of $\Delta_{\mathrm{o}}$ determined from the difference of the maxima of the $\mathrm{A}$ doublet peak is about $1.4 \mathrm{eV}$ for $\mathrm{BiFeO}_{3}$, which is consistent with the experimental values reported recently by other authors $\left[{ }^{77,90}\right]$, while the electronic transition from $e_{g}$ to $t_{2 g}$ orbitals in $\mathrm{Bi}_{0.7} \mathrm{~Tb}_{0.3} \mathrm{FeO}_{3}$ is decreased to about $1.1 \mathrm{eV}$. The reduced value of $\Delta_{o}$ will result in a higher spin state, which is accompanied by a reduced 
hybridization of the $\mathrm{O} 2 p$ and Fe $3 d$ states. This result is in agreement with our ELNES observations and also with the NEXAFS studies of Borisevich et al. $\left[{ }^{83}\right]$ for Sm-substituted $\mathrm{BiFeO}_{3}$. As shown in the inset of Fig. $7 \mathrm{~b}$, the intensity of the A double peak reduces with increasing $\mathrm{Tb}$ concentration, and simultaneously the relative intensity ratio of the peaks decreases with a progressive lowering of the intensity of the high-energy side peak (the $e_{g}$ peak). It is suggested that the reduction of the degree of the $\mathrm{O} 2 p-e_{g}$ hybridization is predominantly responsible for the observed composition-driven decreasing of the Fe $\mathrm{K}$ edge A peak. On the other hand, the B peak increases with increasing Tb content. Similar trends in the evolution of the A and B spectral peaks have been reported for analogous compounds modified with Sm $\left[{ }^{91}\right]$ and $\mathrm{La}\left[{ }^{86}\right]$ and the converse effects in the $1 s-3 d$ and $1 s-4 p$ transitions were explained by the reduced mixing of $3 d$ and $4 p$ levels. Lee et al. $\left[{ }^{92}\right]$ reported the reversed spectral behavior of the $\mathrm{A}$ and $\mathrm{B}$ peaks in epitaxial $\mathrm{BiFeO}_{3}$ thin films and the enhanced hybridization of the $\mathrm{Fe} 3 d$ and $4 p$ orbitals was attributed to the increased in-plane compressive stress in the films. They showed, using the EXAFS spectra, that due to a dissimilar substrate the interatomic distance of the first-shell $\mathrm{Fe}-\mathrm{O}$ along the out-of-plane is larger than that along the in-plane direction, which causes a strong in-plane compressive distortion in the films. Also, an increase in the number of oxygen-vacancy-related distortions of the $\mathrm{FeO}_{6}$ octahedra has been attributed to the increased number of the $1 s-3 d$ dipole-forbidden transitions in $\mathrm{Ca}\left[{ }^{93}\right]$ and $\mathrm{Ta}\left[{ }^{94}\right]$ doped $\mathrm{BiFeO}_{3}$ ceramics. Our XRD analysis showed that the difference in the Rietveld refined bond distance between $\mathrm{Fe}-\mathrm{O} 1$ and $\mathrm{Fe}-\mathrm{O} 2$ reduces significantly upon the substitution of $\mathrm{Tb}$ for $\mathrm{Bi}$ at the $\mathrm{A}$ site of perovskite lattice. Thus, the $\mathrm{Fe}$ ion moves in the $\mathrm{FeO}_{6}$ cage to a more symmetric position, making the octahedron less distorted. This structural effect of $\mathrm{Tb}$ substitution on local $\mathrm{Fe}$ environment was confirmed by the EXAFS results. Fig. S5 (Suppl.) shows the radial distribution function obtained by Fourier transformation of the EXAFS $\chi(\mathrm{k})$ function into real space for pure and $\mathrm{Tb}$-doped $\mathrm{BiFeO}_{3}$. As can be seen, the two distinct peaks corresponding to the first-shell Fe-O bonds come closer to each other as the amount of Tb increases. These results are consistent with the concept of metal-oxygen covalent bonding in the transition metal oxides $\left[{ }^{69,77}\right]$ and perovskites $\left[{ }^{95,96}\right]$. In these materials, a competition between Jahn-Teller distortion and charge disproportion gives rise to the effect of covalent bonding of the oxygen states to the unoccupied $3 d / 4 f$ states of transition metals. With the changing hybridization between the $\mathrm{B}$ site metal atoms and oxygen atoms, the Jahn-Teller distortion of the oxygen octahedron with metal atom in central position changes. Thus, it is reasonable to claim that the observed structural changes of the $\mathrm{FeO}_{6}$ octahedron in $\mathrm{Bi}_{1-\mathrm{x}} \mathrm{Tb}_{\mathrm{x}} \mathrm{FeO}_{3}$ reflect the changes in hybridization between the $\mathrm{Fe}$ ion and $\mathrm{O}$ cation caused by adding $\mathrm{Tb}$. The reduced degree of the $\mathrm{Fe} 3 d-4 p$ 
orbital mixing (from the NEXAFS Fe $\mathrm{K}$ edge spectra), as well as a weaker Fe $3 d-\mathrm{O} 2 p$ hybridization (from the ELNES O K and Fe L edges and confirmed by the XANES Fe K preedge peak evolution) in $\mathrm{Tb}$-doped $\mathrm{BiFeO}_{3}$ samples, can be directly linked with the reduced $\mathrm{Fe}$ $\mathrm{O}$ bond anisotropy of the oxygen octahedra.

A correlation between the $3 d-4 p$ hybridization and magnetization in $\mathrm{La}$ substituted $\mathrm{BiFeO}_{3}$ has been proposed by J. - H. Lee et al. ${ }^{[86}$. They examined the orbital-resolved density of states (DOS) and found that the substitution by La ions increases the number of occupied states in the Fe $3 d$ orbital. Considering that the total magnetic moment in these weak ferromagnets is predominantly produced by the $\mathrm{Fe}^{3+}$ ions, they predicted from the DFT calculations that the net magnetization will increase as long as the $3 d-4 p$ orbitals overlap is suppressed, thus, with the increase in the number of occupied states in the Fe $3 d$ orbital. In addition, they found that the enhancement of macroscopic magnetization is triggered by the net canted moment which increases due to a progressive canting of antiferromagnetically ordered Fe spins. It was also demonstrated that the net canted moment appears in $\mathrm{La}$ doped $\mathrm{BiFeO}_{3}$ within a mirror hexagonal (110)h plane (crystal $c$-axis), irrespective of the doping. It can, thus, be inferred that the weakening of the $3 d-4 p$ hybridization is responsible for the observed increase in magnetization in $\mathrm{Tb}$-doped $\mathrm{BiFeO}_{3}$ samples. However, the reduced degree of the $\mathrm{Fe} 3 d-\mathrm{O} 2 p$ hybridization due to the Tb substitution cannot be ruled out from consideration as well because the Dzyaloshinskii-Moriya interaction, which is responsible for the canting of the magnetic moments in $\mathrm{BiFeO}_{3}\left[{ }^{97}\right]$, is closely related to the super-exchange $\mathrm{Fe}-\mathrm{O}-\mathrm{Fe}$ magnetic interaction, which in turn is known to be very sensitive to small structural changes $\left[{ }^{6}\right]$. As shown by the NEXAFS and EXAFS spectra analyses, local distortions of the $\mathrm{FeO}_{6}$ octahedron such as variations in the $\mathrm{Fe}-\mathrm{O}$ distance and octahedral tilt (simultaneous increase in the relative intensity of the B peak and decrease in the C peak, see Ref. 85 for details) accompany the $\mathrm{Tb}$ substitution-induced suppression of the orbital overlap between the $\mathrm{Fe} 3 d$ and $\mathrm{O} 2 p$ states.

To further investigate the local bonding environment of $\mathrm{Fe}$ atoms in $\mathrm{Tb}$-doped $\mathrm{BiFeO}_{3}$ compounds, ab initio XANES calculations of the $\mathrm{Fe}^{3+}$ ion in the $R 3 c$ and Pnma structures were carried out using FEFF 8.1 and Viper codes. The crystallographic cluster/sphere with 5 A radius around the absorber Fe atom (Suppl., Fig. S6a) was used for the self-consistent calculations and XANES spectra simulations. The full multiple scattering (FMS) calculations included all possible paths within a large cluster radius, typically higher than $8 \AA$ A. Figs. S6b and S6c (in Suppl. material) show the calculated XANES spectra for the $\mathrm{x}=0$ and $\mathrm{x}=0.15$ samples. It can be clearly seen that the simulated spectra mostly reproduce the main features of the experimental data, in particular, those close to the absorption edge; an indication of the 
resonances from the local atomic arrangement of the $R 3 c$ structure in pure $\mathrm{BiFeO}_{3}$ and prevailing orthorhombic arrangement of the nearest neighbor atoms around $\mathrm{Fe}$ in the $\mathrm{Bi}_{0.8} \mathrm{~Tb}_{0.2} \mathrm{FeO}_{3}$ sample. More precise $a b$ initio calculations with the input data from high resolution synchrotron XRD and neutron diffraction measurements and upon consideration of all the electronic transitions associated with XAFS measurements are planned to be carried out in order to fit the measured fine structures, including higher coordination spheres with higher accuracy.

The effect of the Fe $3 d$ - O $2 p$ hybridization on macroscopic magnetization of the Tb-doped $\mathrm{BiFeO}_{3}$ ceramics can be interpreted also in terms of evolution of filled $3 d$ levels. In $\mathrm{BiFeO}_{3}$ and $\mathrm{BiFeO}_{3}$-related magnetoelectric compounds, a $G$-type antiferromagnetic order with the Fe spins coupled ferromagnetically within the hexagonal $(001)_{h}$ planes and antiferromagneticaly along the hexagonal $[001]_{\mathrm{h}}$ direction $\left[{ }^{98,99,100}\right]$ occurs, in conformity with both Pauli exclusion principles and Goodenough-Kanamori-Anderson rules, because of the strong exchange interaction between neighboring Fe half-filled $e_{g}$ orbitals through oxygen $2 p \sigma$-bonding.[ $\left.{ }^{101}\right]$ This chemical bonding generates a mixture of the electronic $\mathrm{Fe} 3 d^{5}$ and $\mathrm{Fe} 3 d^{6} \underline{\mathrm{L}}$ states ( $\underline{\mathrm{L}}$ is a ligand hole state generated through a charge transfer from $\mathrm{O} 2 p^{6}$ to $\mathrm{Fe} 3 d^{5}$ ). Upon $\mathrm{Tb}$ substitution, the hybridization of unoccupied Fe $3 d$ and $\mathrm{O} 2 p$ states was shown to be reduced, as demonstrated in Fig. $7 \mathrm{~b}$ by the progressive decreasing of the relative intensity of the A doublet peak accompanied by a variation in the peaks intensities ratio. The $t_{2 g}$ peak increases, while that corresponding to the number of unoccupied $e_{g}$ states decreases with increasing $\mathrm{Tb}$ concentration. P. Trivedi et al $\left.{ }^{102}\right]$ observed similar converse effects of the doublets for the $\mathrm{Fe}$ $\mathrm{L}_{2,3}$ edge spectra of $\mathrm{Er}, \mathrm{La}$ and $\mathrm{Pr}$ modified $\mathrm{BiFeO}_{3}$ and attributed it to the rare earth substitution induced increase in the number of occupied the Fe $3 d^{5}$ states at the expense of the Fe $3 d^{6} \underline{\mathrm{L}}$ states. Again, analogous to the $3 d-4 p$ orbital mixing effect, the weaker Fe $3 d-\mathrm{O} 2 p$ hybridization accompanied by the increased number of occupied states in the Fe $3 d$ orbital may partly also contribute to the enhanced magnetization of $\mathrm{Tb}$-doped $\mathrm{BiFeO}_{3}$.

Recent DFT studies $\left[{ }^{103}\right]$ indicate that the presence of rare earths can slightly change the local magnetic moment on the $\mathrm{Fe}$ sites due to the hybridization of the $\mathrm{Fe} 3 d$ - and $\mathrm{O} 2 p$-states with rare earth localized $4 f$ states. It was shown that the magnetization of rare earth doped $\mathrm{BiFeO}_{3}$ increases with increasing number of $4 f$ electrons. Therefore, in our Tb-doped samples the $f$ - $d$ exchange interaction can partly contribute to the observed increase in magnetization, at cryogenic temperatures at the least.

\section{Low-temperature magnetic properties}


Comparison of the field dependences of magnetization measured at 300 and 5 K (Fig. 5a and Fig. 5b, respectively) for the pure and $\mathrm{Tb}$-doped $\mathrm{BiFeO}_{3}$ samples subjected to a maximal magnetic field of $\pm 5 \mathrm{~T}$ shows that the substitution-driven enhancement of the net magnetic moment is more prominent at lower temperatures, where terbium atoms with their own large magnetic moments make an important contribution to magnetization. The $\mathrm{M}-\mathrm{H}$ hysteresis loops recorded at $\mathrm{T}=5 \mathrm{~K}$ are well developed with significantly higher magnetization values than those obtained at room temperature. Such a behavior implies that the molecular field of the ordered $\mathrm{Fe}^{3+}$ ions magnetizes $\mathrm{Tb}^{3+}$ ions via $4 f-3 d$ exchange coupling, aligning their magnetic moments parallel with respect to a weak ferromagnetic component of the canted Fe subsystem to provide a significant enhancement of the magnetization at $5 \mathrm{~K}$. Similar observations on the enhanced magnetization at low temperatures have been reported for rare-earth substituted ferrites, such as $\mathrm{TbFeO}_{3}$ with a perovskite type orthorhombic Pnma structure.[ $\left.{ }^{24}\right]$ Substitutionintroduced antiferromagnetic $\mathrm{Tb}^{3+}-\mathrm{Fe}^{3+}$ and $\mathrm{Tb}^{3+}-\mathrm{Tb}^{3+}$ spin interactions in the orthorhombic Pnma phase are expected to weaken the antiferromagnetic interactions between the $\mathrm{Fe}^{3+}$ ions. In particularly, the former coupling between the $\mathrm{Fe}^{3+}$ and $\mathrm{Tb}^{3+}$ magnetic moments through the intervening $\mathrm{O}^{2-}$ ions in $90^{\circ}$ position may suppress the magnetic ordering at higher temperatures to such an extent that the antiferromagnetic Néel temperature of $\mathrm{BiFeO}_{3}$ is decreased, as observed by DSC (Fig. 4). The reduction in the coercive field at low temperature, as compared with the room temperature value for all the compositions studied, can be explained by the presence of magnetoelectric coupling in these multiferroic systems. [ $\left.{ }^{104,105,106}\right]$ Ruette et al. $\left[{ }^{100}\right]$ have proposed that the interplay between magnetic order and electrical order parameters produces a competing interaction, which acts to decrease the effective magnetic anisotropy in magnetoelectrics. A similar reduction in coercivity at low temperatures has been previously reported for Dy-, Sm- and Gd-doped $\mathrm{BiFeO}_{3}$ ceramics.[ ${ }^{17,55,106}$ ]

To further investigate the magnetic properties and evaluate the development of the positive $4 f$ $3 d$ magnetism upon doping, the temperature dependence of magnetization of Tb-substituted $\mathrm{BiFeO}_{3}$ ceramics was measured over the temperature range $5-300 \mathrm{~K}$ in the presence of an externally applied magnetic field. Fig. 8a shows the field cooling/field heating (FC/FH) magnetization vs. temperature plots for the SPS'ed samples of $\mathrm{Bi}_{1-\mathrm{x}} \mathrm{Tb}_{\mathrm{x}} \mathrm{FeO}_{3}(\mathrm{x}=0,0.05,0.10$, 0.20 and 0.30 ) subjected to an external magnetic field of 100 Oe. Pure $\mathrm{BiFeO}_{3}$ exhibits a conventional antiferromagnetic behavior in the temperature range of $120 \mathrm{~K}-300 \mathrm{~K}$ for both the field-cooling and field-heating regimes: the small but non-zero magnetization decreases with the decrease in the temperature and vice versa (the inset of Fig. 8a). The character of the magnetic response is determined by the superexchange coupling between iron magnetic 
moments $\left(\mathrm{Fe}^{3+}-\mathrm{O}^{2-}-\mathrm{Fe}^{3+}\right)$. Below $20 \mathrm{~K}$, a steep increase in the $\mathrm{FC}$ magnetization values towards $5 \mathrm{~K}$ and sudden drop in the $\mathrm{FH}$ magnetization when heating the sample from $5 \mathrm{~K}$ reflects the presence of residual magnetic impurities in the $\mathrm{x}=0$ sample. Lebegue et al. $\left[{ }^{107}\right]$ have demonstrated that even small amounts $(\sim 1 \mathrm{~mol} \%)$ of defect $\mathrm{Fe}^{3+}$ ions, for instance due to poor quality of starting materials or presence of the sillenite-type impurity phase in sintered $\mathrm{BiFeO}_{3}$ materials, can produce a Curie-like upturn at low temperatures. Our XRD measurements showed a tiny amount of $\mathrm{Bi}_{2} \mathrm{Fe}_{4} \mathrm{O}_{9}-$ like phase to be present in pure $\mathrm{BiFeO}_{3}$ ceramics. However, this secondary phase is not ferroelectric, undergoes an antiferromagnetic-to-paramagnetic

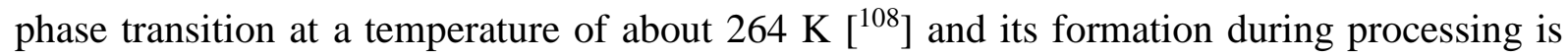
eliminated by $\mathrm{Tb}$ doping, which means that its presence in pure $\mathrm{BiFeO}_{3}$ has no influence on the main results and conclusions of this work. Both the FC and FH magnetization curves for the $\mathrm{x}$ = 0 sample show reversible behavior at temperatures below $70 \mathrm{~K}$. A small thermo-magnetic irreversibility observed at higher temperatures is likely caused by the relaxation processes associated with a thermal response of the $\mathrm{Bi}_{2} \mathrm{Fe}_{4} \mathrm{O}_{9}$-type impurity. The onset of an antiferromagnetic ordering in $\mathrm{Bi}_{2} \mathrm{Fe}_{4} \mathrm{O}_{9}$ is thought to be responsible for an anomaly observed in the FC and FH M-T curves at temperatures between 250 and $270 \mathrm{~K}$ (see the inset of Fig. 7a). Similar trends in ZFC and FC magnetization curves were reported for single crystals $\left[{ }^{109}\right]$ and nanoparticles $\left[{ }^{110}\right]$ of $\mathrm{BiFeO}_{3}$. On the other hand, there is no evidence for spin-glass behavior or spin reorientation transitions in the measured temperature dependent magnetization, as previously reported for $\mathrm{BiFeO}_{3}\left[{ }^{109}\right]$.

Upon doping, the temperature dependence of the magnetization changes its character from antiferromagnetic to ferromagnetic with a weak magnetic moment. Fig. 8b displays the representative data for the $\mathrm{x}=0.15$ sample. The FC susceptibility $(\chi=\mathrm{M} / \mathrm{H})$ gradually increases with decreasing the temperature from 300 to $50 \mathrm{~K}$ due to local clustering of spins, and then, when further cooled down to $5 \mathrm{~K}$, it sharply increases following a modified Curie-Weiss law $\left[{ }^{111}\right]$ :

$$
\chi=\frac{C}{T-T_{C W}}+\chi_{0}
$$

, where $\mathrm{T}$ is the temperature and $\mathrm{T}_{\mathrm{CW}}$ is the critical temperature. The constants $C$ and $\chi_{0}$ represent the material-related Curie constant and the temperature-independent magnetic contributions, respectively. When $\mathrm{T}_{C W}$ is positive, the predominant interactions are ferromagnetic even though long-range magnetic ordering may not occur. A negative value of $\mathrm{T}_{\mathrm{CW}}$ implies the dominating role of the antiferromagnetic interactions. Fitting of the $\chi(\mathrm{T})$ curve gives for $\mathrm{Bi}_{0.85} \mathrm{~Tb}_{0.15} \mathrm{FeO}_{3}$ the value of $\mathrm{T}_{\mathrm{CW}} \approx-4.5 \mathrm{~K}$, indicative of the predominance of 
antiferromagnetic interactions. For the samples with doping levels of 0.05, 0.10, 0.20, 0.25 and 0.30, the calculated Curie-Weiss temperatures are -5.5, -5.0, -3.6, -3.6 and -3.1 K, respectively, confirming antiferromagnetic ordering of iron ions in all these structures. Moreover, $\mathrm{T}_{\mathrm{CW}}$ slightly increases with increasing doping level, which suggests an enhanced exchange interaction between the transition metal ions. The ordered magnetic moment determined from the thermal data of the $\mathrm{x}=0.15$ sample $(\mu=2.827 \sqrt{ } C)$ is about $3.96 \mu_{B}\left(\mu_{B}\right.$ is the Bohr magneton), which is in accordance with the value $\mu=4.0(3) \mu_{B}$ obtained by neutron scattering on similar compositions by Saxin et al. $\left[{ }^{27}\right]$.

\section{Conclusions}

In summary, dense ceramic samples of the $\mathrm{Bi}_{1-\mathrm{x}} \mathrm{Tb}_{\mathrm{x}} \mathrm{FeO}_{3}$ solid-solution system with $\mathrm{x}$ varying from 0 to 0.30 were prepared by SPS technique and their structural and magnetic properties were systematically studied with respect to the added $\mathrm{Tb}$ content. The XRD study complemented by Raman spectroscopy data revealed that the A-site terbium substitution causes the transformation of an initial rhombohedral structure (space group $R 3 c$ ) to an orthorhombic (Pnma) phase. The two phases with a variable R3c/Pnma weight ratio were found to coexist in samples over a wide concentration range $0.10 \leq \mathrm{x} \leq 0.20$. Results of detailed structural analyses on these biphasic compositions suggest that the polar-to-nonpolar phase transition is predominantly driven by tilting of oxygen octahedra. The observed substitution-induced suppression of low-frequency $R 3 c E+A_{l}$ Raman active modes (broadening, damping and blueshifting) in the doped samples indicates the weakening of long-range ferroelectric order due to dilution of $\mathrm{Bi} 6 s^{2}$ lone pairs by $\mathrm{Tb}$ doping. The reduced degree of the $\mathrm{Bi}-\mathrm{O}$ hybridization in Tb-doped samples was demonstrated by the ELNES spectroscopy. The progressive loss of ferroelectricity with increasing Tb content was also confirmed by DSC measurements, which showed a decreasing Curie point with increasing $\mathrm{Tb}$ substitution. SQUID magnetometry revealed the existence of the weak room-temperature ferromagnetism in $\mathrm{Bi}_{1-\mathrm{x}} \mathrm{Tb}_{\mathrm{x}} \mathrm{FeO}_{3}$ ceramic series. The appearance of a weak ferromagnetic moment even at small amounts of $\mathrm{Tb}(\mathrm{x}=0.05)$ and the enhancement of the net magnetization on further doping was found to nicely correlate with the unveiled structural evolution. The increased antiphase octahedral tilt accompanying the substitution-induced lattice distortion resulted not only in the structural transformation, but provoked also the transition from a spatially modulated canted spin structure to a homogeneous commensurate antiferromagnetic ordering of spins. It is suggested that the dominant mechanism responsible for the enhanced magnetic moment originates from the progressive 
Dzyaloshinskii-Moryia interaction driven by the structural $R 3 c$-to-Pnma phase transition. The results of the STEM-ELNES and XAFS studies at the O K, Fe L and Fe K edges of the $\mathrm{Bi}_{1}$ ${ }_{x} \mathrm{~Tb}_{\mathrm{x}} \mathrm{FeO}_{3}$ ceramics indicate that the changes in the electronic structure (e.g., the weakening of both the $3 d-4 p$ and $\mathrm{Fe} 3 d-\mathrm{O} 2 p$ hybridizations) by the Tb doping may result in the enhanced macroscopic magnetization due to local structural distortions in the $\mathrm{FeO}_{6}$ octahedron. The SPS'ed sample exhibiting a ferroelectric ordering and, at the same time, the highest values of the magnetization at room temperature corresponded to a composition with $\mathrm{x} \approx 0.20$. At low temperatures, a significant increase in the net magnetization was observed and ascribed to an indirect magnetic interaction (a positive $f$ - $d$ exchange) between the introduced $\mathrm{Tb}^{3+}$ ions and the canted antiferromagnetic Fe subsystem.

The results of the present work are of importance for understanding the phenomena of the composition-driven phase transitions and weak ferromagnetic state in rare-earth-substituted $\mathrm{BiFeO}_{3}$ multiferroics. A comprehensive study of structural and multiferroic properties of spark plasma processed ceramics within the $\mathrm{Bi}_{1-\mathrm{x}} \mathrm{Tb}_{\mathrm{x}} \mathrm{FeO}_{3}$ series enabled to identify the composition which offers a phase-pure room-temperature multiferroic with improved magnetic properties.

\section{Acknowledgement}

This work has been supported by the Grant Agency of the Slovak Academy of Sciences through grants No. 2/0057/14 and 2/059/17 and by the Slovak Research and Development Agency through the grant No. APVV-15-0115. 


\section{Figures captions}

Figure 1. (a) Room-temperature X-ray diffraction patterns of $\mathrm{Bi}_{1-x} \mathrm{~Tb}_{x} \mathrm{FeO}_{3}(0 \leq \mathrm{x} \leq 0.30)$ ceramics prepared by SPS. The main peak from the $\mathrm{Bi}_{2} \mathrm{Fe}_{4} \mathrm{O}_{9}$ impurity phase is indicated by asterisk (*) for the $\mathrm{BiFeO}_{3}(\mathrm{x}=0)$ sample. (b) and (c) display the enlarged parts of the patterns to demonstrate structure evolution on doping.

Figure 2. The substitution-induced variation in the primitive cell volume of $\mathrm{Tb}$-substituted $\mathrm{BiFeO}_{3}$ ceramics.

Figure 3. Raman spectra of the SPS processed $\mathrm{Bi}_{1-\mathrm{x}} \mathrm{Tb}_{\mathrm{x}} \mathrm{FeO}_{3}(0 \leq \mathrm{x} \leq 0.30)$ ceramics (collected at room remperature). The inset demonstrates the appearance and evolution of the phonon mode at $\approx 620 \mathrm{~cm}^{-1}$.

Figure 4. (a) Specific-heat measurements of $\mathrm{BiFeO}_{3}(\mathrm{x}=0)$ on heating and cooling. The inset shows the Néel temperature of the antiferromagnetic-to-paramagnetic transition. (b) and (c) compare the DSC data obtained for $\mathrm{Bi}_{1-\mathrm{x}} \mathrm{Tb}_{\mathrm{x}} \mathrm{FeO}_{3}(0 \leq \mathrm{x} \leq 0.30)$ ceramics in the heating and cooling regimes, respectively. The temperatures of the magnetic and ferroelectric phase transitions are indicated for each composition.

Figure 5. Magnetic hysteresis loops obtained for the $\mathrm{Bi}_{1-\mathrm{x}} \mathrm{Tb}_{\mathrm{x}} \mathrm{FeO}_{3}(\mathrm{x}=0,0.05,0.15$ and 0.25) samples in a maximum applied field of $5 \mathrm{~T}$ at (a) $300 \mathrm{~K}$ and (b) $5 \mathrm{~K}$. The inset in (a) shows the dependence of the remanent magnetization on addition of $\mathrm{Tb}$ and (b) shows the enlarged loops around zero field.

Figure 6. (a) Atomic resolution RGB composite image of $\mathrm{BiFeO}_{3}$ ([010] view direction). The STEM-HAADF image (blue) is interleaved with STEM-ABF (yellow) image to differentiate atomic positions of $\mathrm{Bi}, \mathrm{Fe}$ and light oxygen atoms. In the enlarged image (inset), cations of $\mathrm{Bi}$ and $\mathrm{Fe}$ are marked by purple and light brown open circles, respectively; $\mathrm{O}$ anions are rounded by red circles. (b) and (c) HAADF images overlaid by the [010] projected crystal structures of the respective $R 3 c$ and Pnma unit cells (insets: $\mathrm{Bi}$ - purple, $\mathrm{Fe}$ - brown, and $\mathrm{O}$ - red circles) for the $\mathrm{BiFeO}_{3}$ and $\mathrm{Bi}_{0.7} \mathrm{~Tb}_{0.3} \mathrm{FeO}_{3}$ samples, respectively. Scale bar is $2 \mathrm{~nm}$. 
Figure 7. (a) The ELNES O K edge spectra, and (b) the NEXAFS Fe K edge spectra of $\mathrm{Bi}_{1-}$ ${ }_{x} \mathrm{~Tb}_{\mathrm{x}} \mathrm{FeO}_{3}(\mathrm{x}=0,0.15$ and 0.30$)$.

Figure 8. (a) Magnetization as a function of temperature for $\mathrm{Bi}_{1-\mathrm{x}} \mathrm{Tb}_{\mathrm{x}} \mathrm{FeO}_{3}(\mathrm{x}=0,0.05,0.10$, 0.20 and 0.30 ) ceramics subjected to a magnetic field of 100 Oe. The inset shows the thermomagnetic data of pure $\mathrm{BiFeO}_{3}$ at cryogenic temperatures with an anomaly at about 260 $\mathrm{K}$ due to the presence of antiferromagnetic $\mathrm{Bi}_{2} \mathrm{Fe}_{4} \mathrm{O}_{9}$. (b) Curie-Weiss behaviour of the magnetic susceptibility of the $\mathrm{Bi}_{0.85} \mathrm{~Tb}_{0.15} \mathrm{FeO}_{3}$ sample. The inset shows the reciprocal susceptibility as a function of temperature. Full squares represent the experimental data, red solid lines are the Curie-Weiss model fits. 

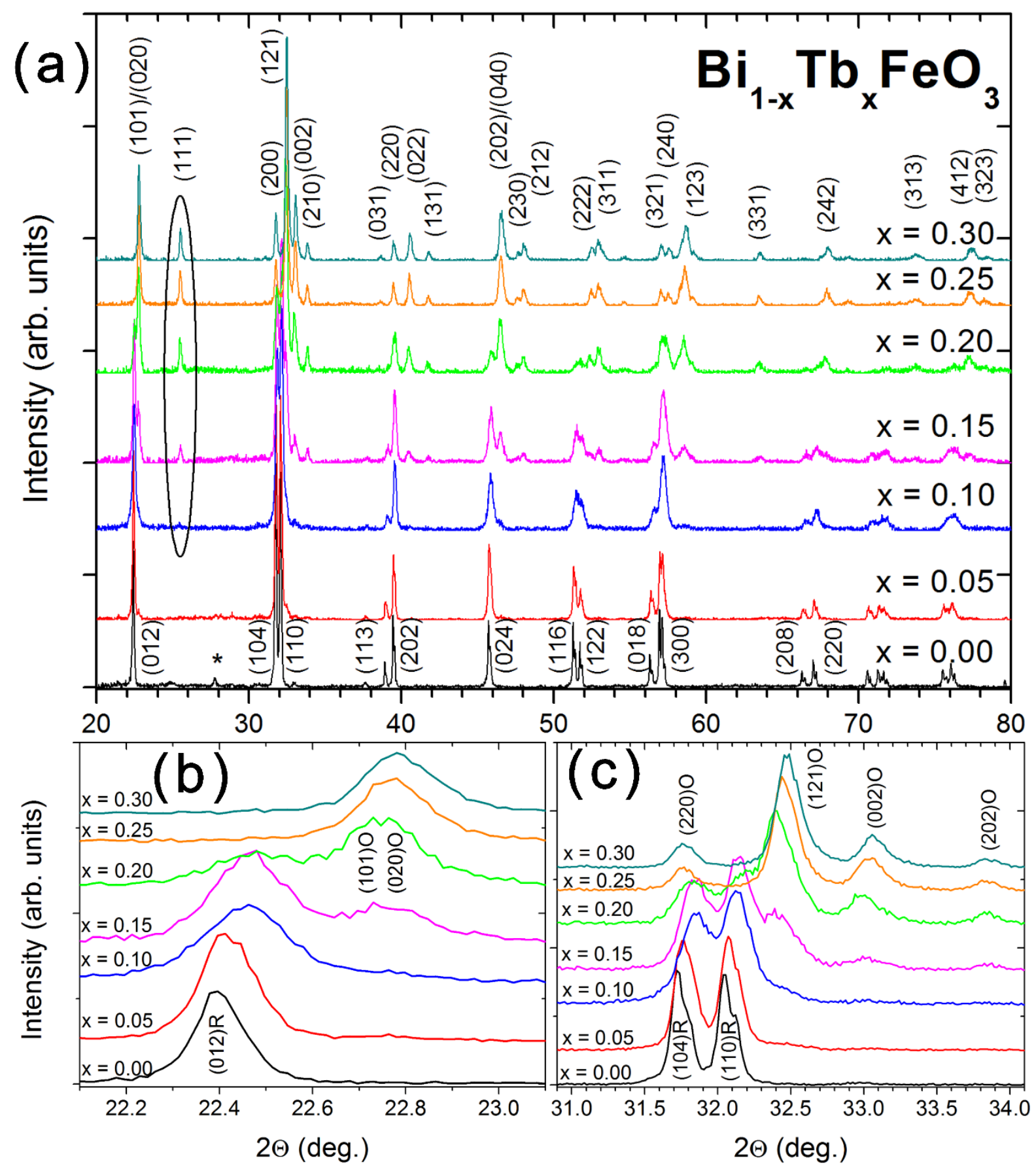

Figure 1. (a) Room-temperature $\mathrm{X}$-ray diffraction patterns of $\mathrm{Bi}_{1-\mathrm{x}} \mathrm{Tb}_{\mathrm{x}} \mathrm{FeO}_{3}(0 \leq \mathrm{x} \leq 0.30)$ ceramics prepared by SPS. The main peak from the $\mathrm{Bi}_{2} \mathrm{Fe}_{4} \mathrm{O}_{9}$ impurity phase is indicated by asterisk (*) for the $\mathrm{BiFeO}_{3}(\mathrm{x}=0)$ sample. $(\mathrm{b})$ and $(\mathrm{c})$ display the enlarged parts of the patterns to demonstrate structure evolution on doping. 


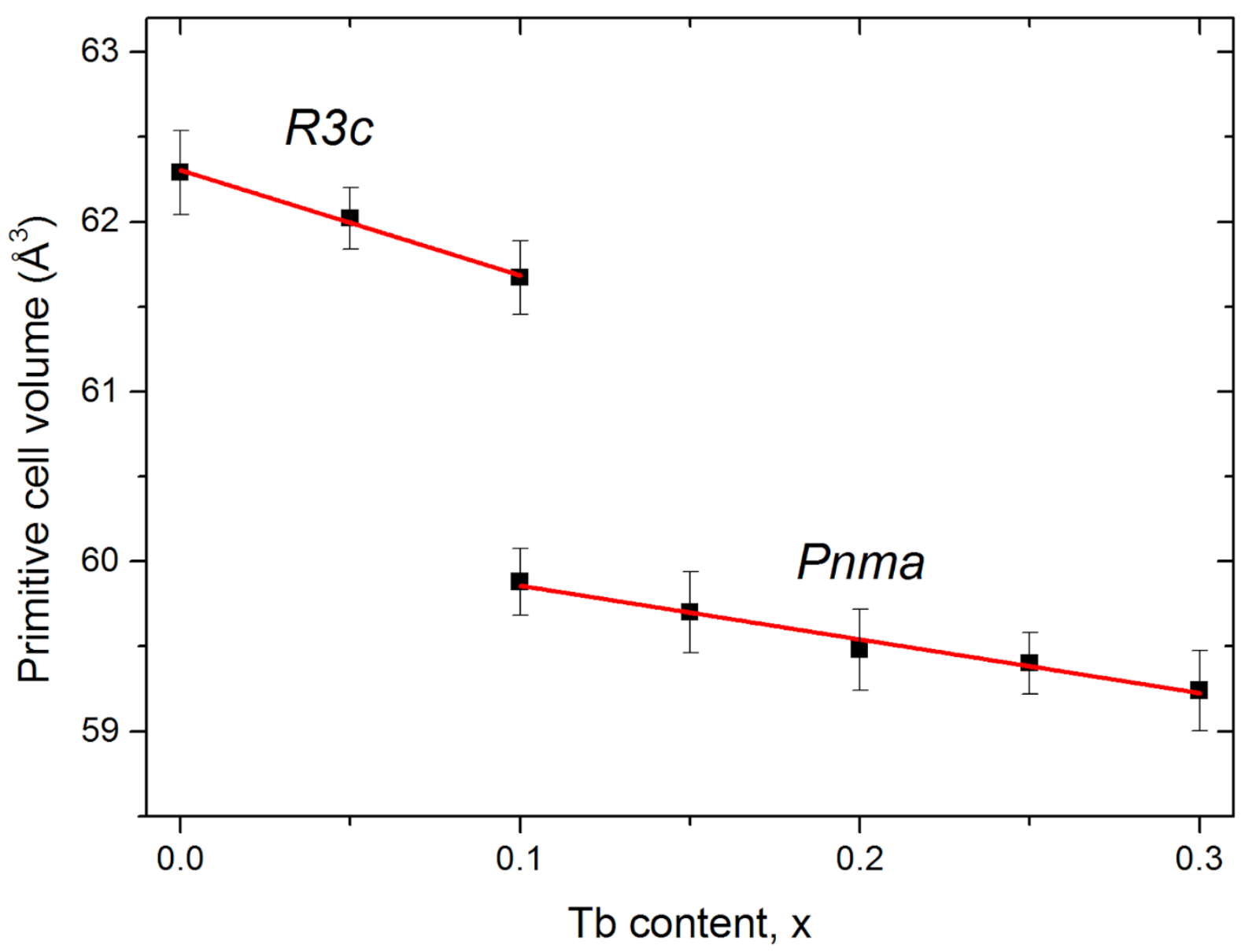

Figure 2. The substitution-induced variation in the primitive cell volume of Tb-substituted $\mathrm{BiFeO}_{3}$ ceramics. 


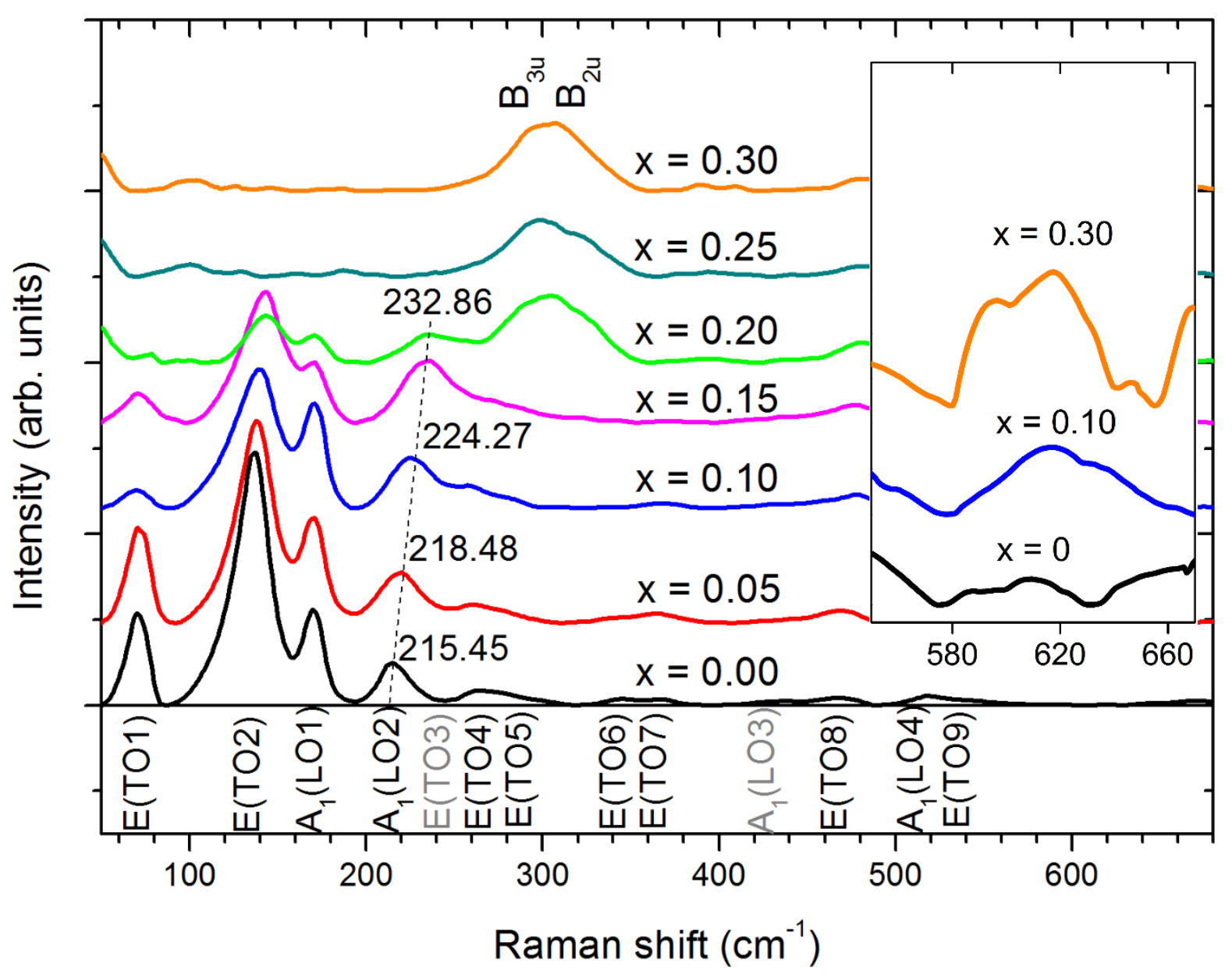

Figure 3. Raman spectra of the SPS processed $\mathrm{Bi}_{1-\mathrm{x}} \mathrm{Tb}_{\mathrm{x}} \mathrm{FeO}_{3}(0 \leq \mathrm{x} \leq 0.30)$ ceramics (collected at room remperature). The inset demonstrates the appearance and evolution of the phonon mode at $\approx 620 \mathrm{~cm}^{-1}$. 

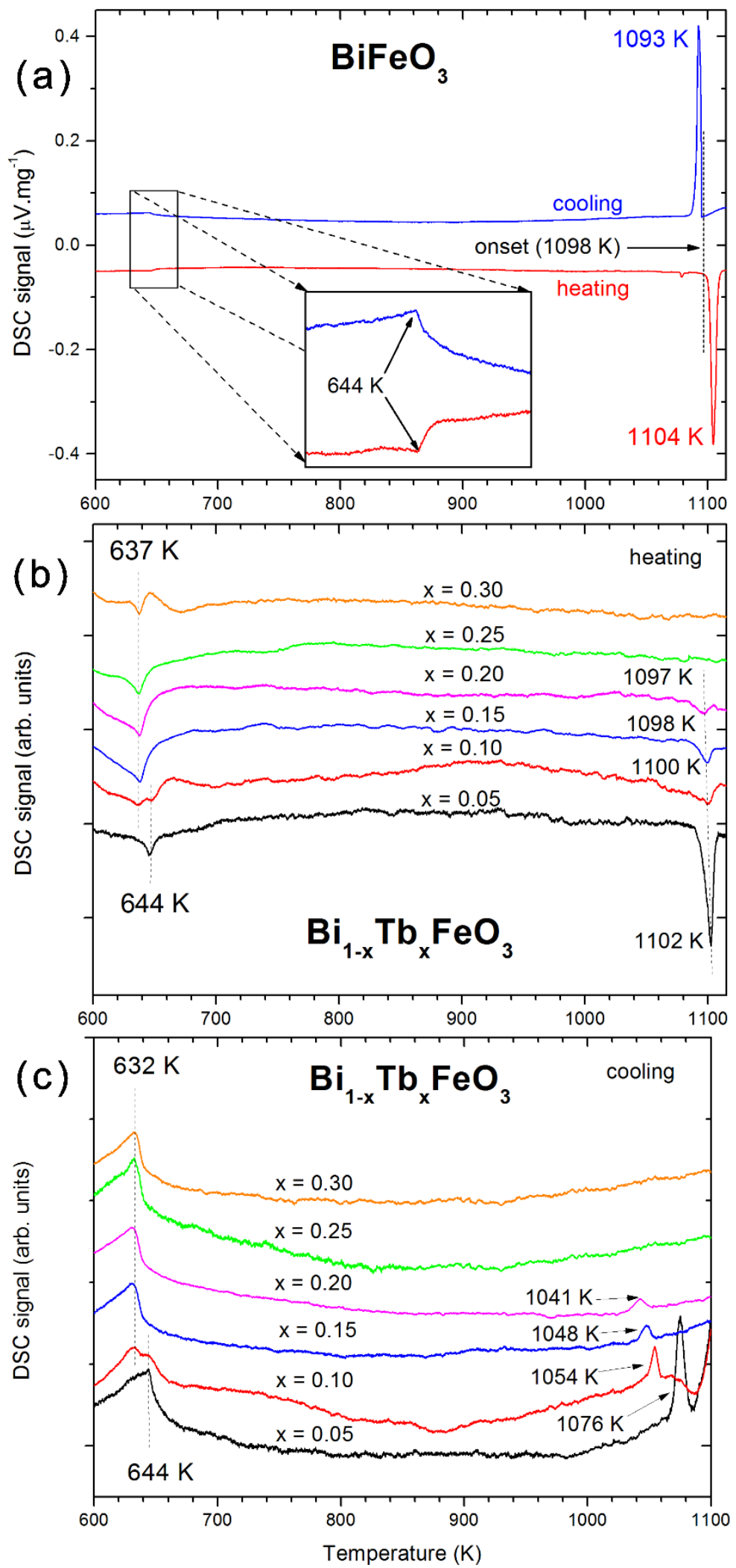

Figure 4. (a) Specific-heat measurements of $\mathrm{BiFeO}_{3}(\mathrm{x}=0)$ on heating and cooling. The inset shows the Neél temperature of the antiferromagnetic-to-paramagnetic transition. (b) and (c) compare the DSC data obtained for $\mathrm{Bi}_{1-\mathrm{x}} \mathrm{Tb}_{\mathrm{x}} \mathrm{FeO}_{3}(0 \leq \mathrm{x} \leq 0.30)$ ceramics in the heating and cooling regimes, respectively. The temperatures of the magnetic and ferroelectric phase transitions are indicated for each composition. 

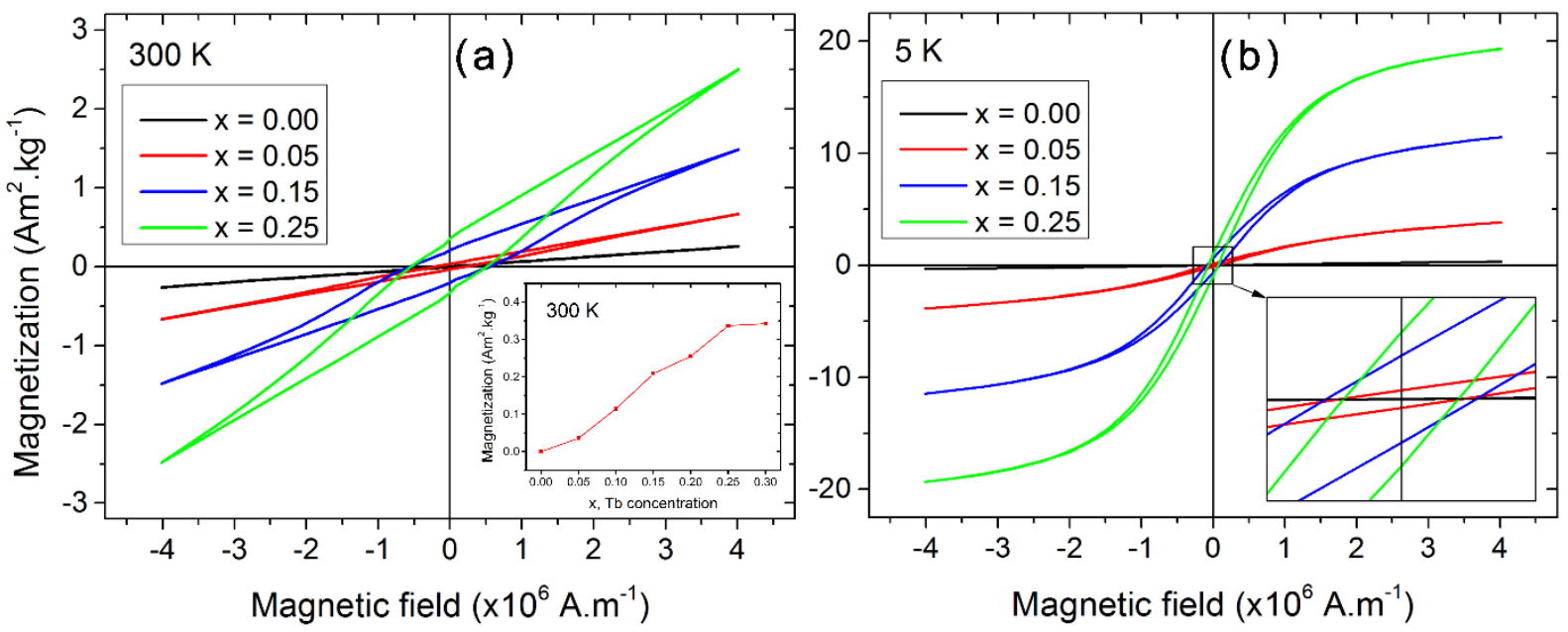

Figure 5. Magnetic hysteresis loops obtained for the $\mathrm{Bi}_{1-\mathrm{x}} \mathrm{Tb}_{\mathrm{x}} \mathrm{FeO}_{3}(\mathrm{x}=0,0.05,0.15$ and 0.25$)$ samples in a maximum applied field of $5 \mathrm{~T}$ at (a) $300 \mathrm{~K}$ and (b) $5 \mathrm{~K}$. The inset in (a) shows the dependence of the remanent magnetization on addition of $\mathrm{Tb}$ and in (b) shows the enlarged loops around zero field. 

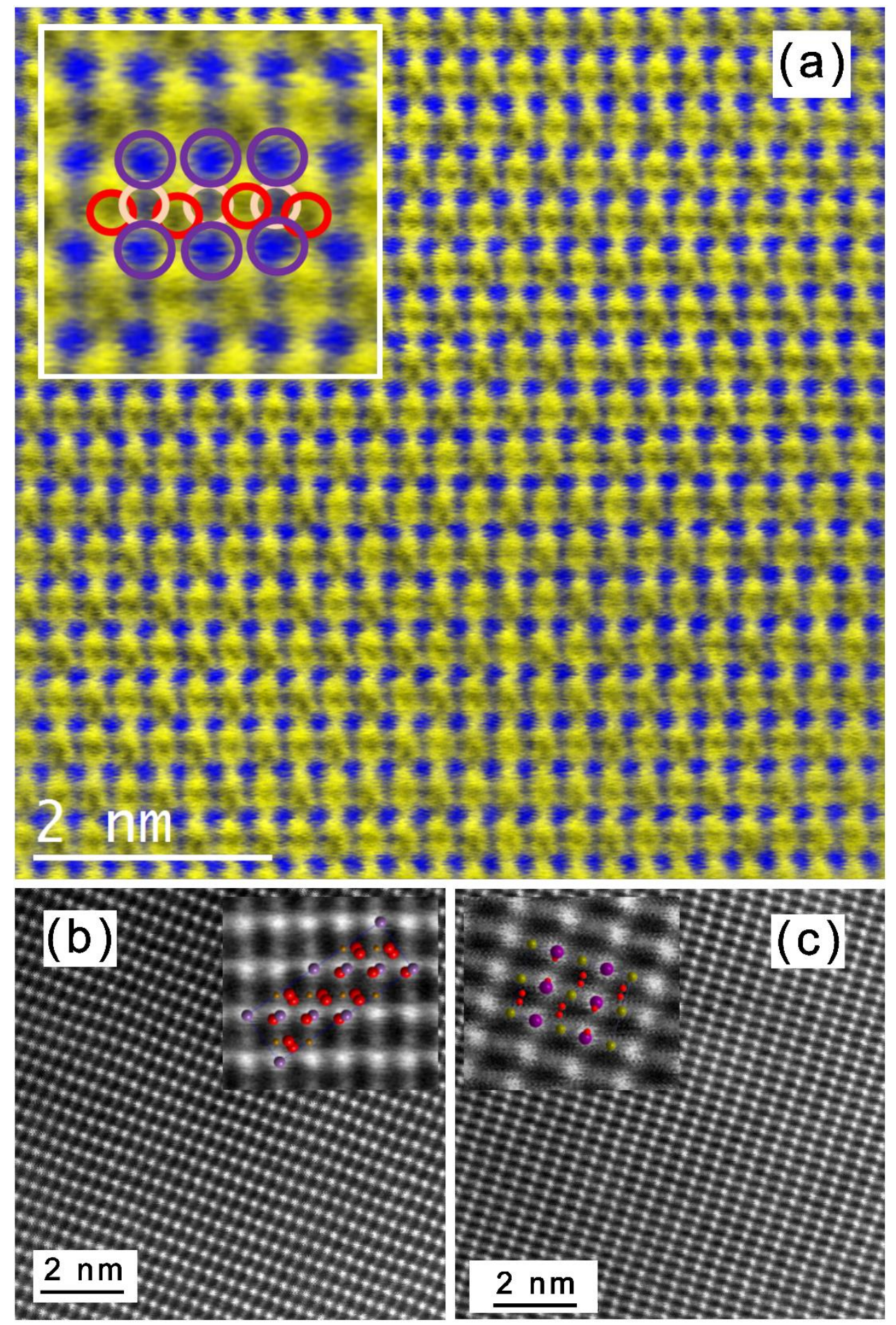

Figure 6. (a) Atomic resolution RGB composite image of $\mathrm{BiFeO}_{3}$ ([010] view direction). The STEM-HAADF image (blue) is interleaved with STEM-ABF (yellow) image to differentiate atomic positions of $\mathrm{Bi}, \mathrm{Fe}$ and light oxygen atoms. In the enlarged image (inset), cations of $\mathrm{Bi}$ and $\mathrm{Fe}$ are marked by purple and light brown open circles, respectively; $\mathrm{O}$ anions are rounded by red circles. (b) and (c) HAADF images overlaid by the [010] projected crystal structures of the respective $R 3 c$ and Pnma unit cells (insets: $\mathrm{Bi}$ - purple, $\mathrm{Fe}$ - brown, and $\mathrm{O}$ - red circles) for the $\mathrm{BiFeO}_{3}$ and $\mathrm{Bi}_{0.7} \mathrm{~Tb}_{0.3} \mathrm{FeO}_{3}$ samples, respectively. Scale bar is $2 \mathrm{~nm}$. 

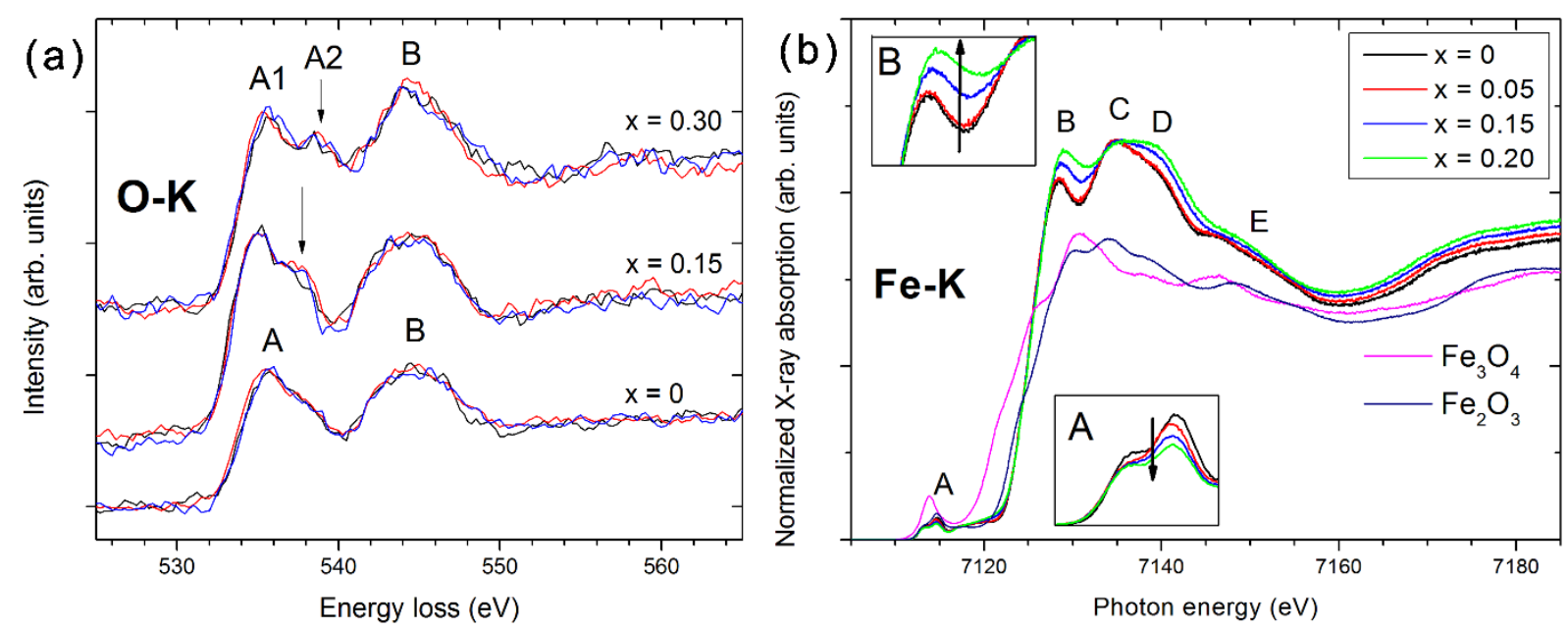

Figure 7. (a) ELNES O K edge spectra (b) NEXAFS Fe K edge spectra of $\mathrm{Bi}_{1-\mathrm{x}} \mathrm{Tb}_{\mathrm{x}} \mathrm{FeO}_{3}(\mathrm{x}=$ $0,0.15$ and 0.30$)$. 

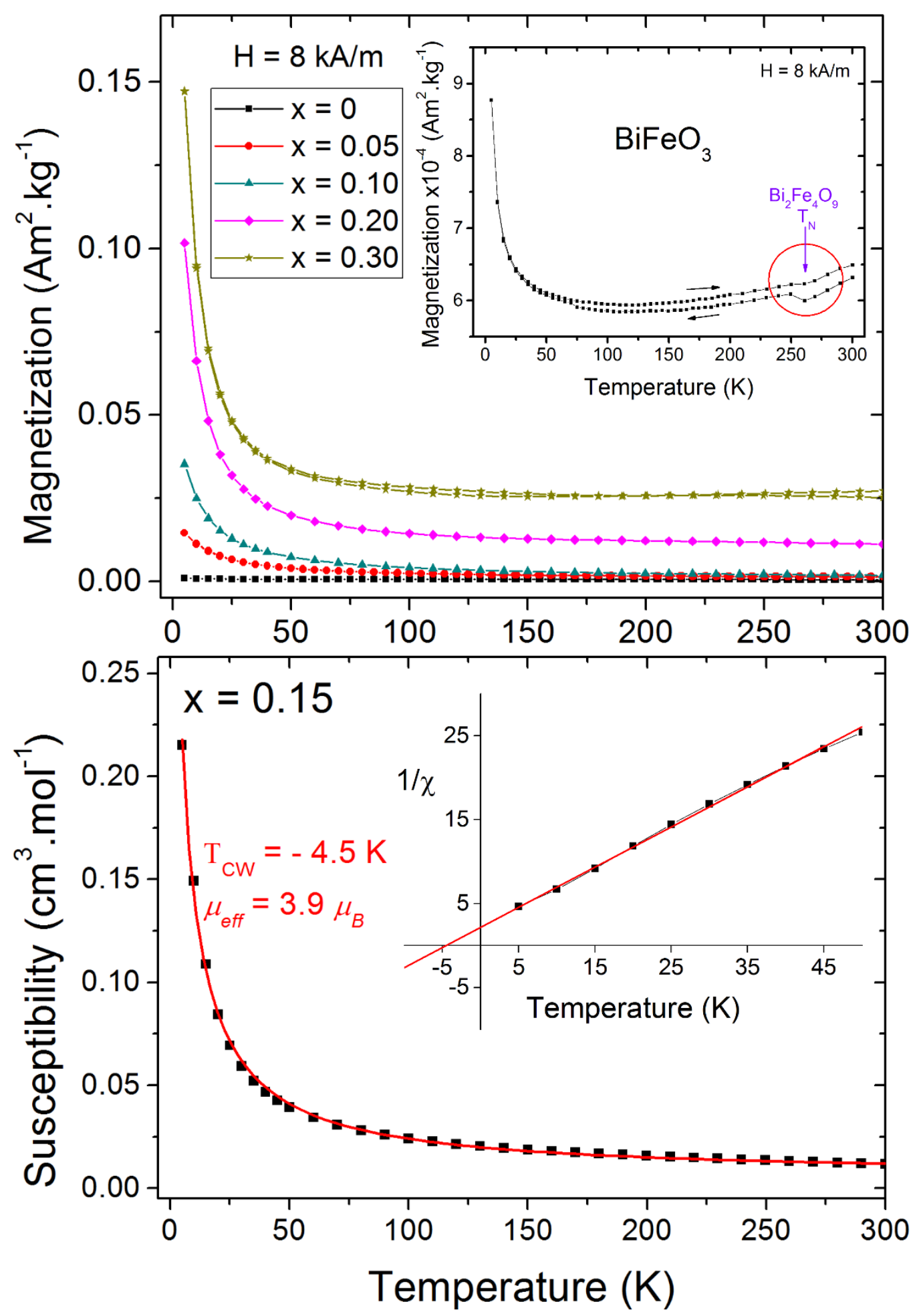

Figure 8. (a) Magnetization as a function of temperature for $\mathrm{Bi}_{1-\mathrm{x}} \mathrm{Tb}_{\mathrm{x}} \mathrm{FeO}_{3}(\mathrm{x}=0,0.05,0.10$, 0.20 and 0.30 ) ceramics subjected to a magnetic field of 100 Oe. The inset shows the thermomagnetic data of pure $\mathrm{BiFeO}_{3}$ at cryogenic temperatures with an anomaly at about 260 $\mathrm{K}$ due to the presence of antiferromagnetic $\mathrm{Bi}_{2} \mathrm{Fe}_{4} \mathrm{O}_{9}$. (b) Curie-Weiss behaviour of the magnetic susceptibility of the $\mathrm{Bi}_{0.85} \mathrm{~Tb}_{0.15} \mathrm{FeO}_{3}$ sample. The inset shows the reciprocal susceptibility as a function of temperature. Full squares represent the experimental data, red solid lines are the Curie-Weiss model fits. 


\section{Supplementary material}

Terbium-induced phase transitions and weak ferromagnetism in multiferroic bismuth ferrite ceramics

Vladimir Koval ${ }^{1}$, Ivan Skorvanek ${ }^{2}$, Juraj Durisin ${ }^{1}$, Giuseppe Viola ${ }^{3,4}$, Alexandra Kovalcikova ${ }^{1}$, Peter Svec jr. ${ }^{5}$, Karel Saksl ${ }^{1}$, and Haixue Yan $^{4}$

${ }^{1}$ Institute of Materials Research, Slovak Academy of Sciences, Kosice, Slovakia

${ }^{2}$ Institute of Experimental Physics, Slovak Academy of Sciences, Kosice, Slovakia

${ }^{3}$ Institute of Materials Physics and Engineering, Torino, Italy

${ }^{4}$ School of Engineering and Materials Science, Queen Mary, University of London, London, United Kingdom, Mile End Road, E1 4NS

${ }^{5}$ Institute of Physics, Slovak Academy of Sciences, Bratislava, Slovakia

Table $\mathrm{S} 1$. The crystal symmetry and refined unit cell parameters of the $\mathrm{Bi}_{1-\mathrm{x}} \mathrm{Tb}_{\mathrm{x}} \mathrm{FeO}_{3}$ samples (the values obtained by Rietveld refinement of the room-temperature XRD data).

\begin{tabular}{llllll}
\hline Sample & Phase & \multicolumn{4}{c}{ Lattice parameters } \\
$\mathrm{x}$ & $(\mathrm{wt} . \%)$ & $a(\AA)$ & $b(\AA)$ & $c(\AA)$ & $V\left(\AA^{3}\right)$ \\
\hline 0 & $R 3 c(98 \%)^{*}$ & $5.5782(0)$ & $5.5782(0)$ & $13.8686(1)$ & $373.73(0)$ \\
0.05 & $R 3 c(99 \%)^{*}$ & $5.5716(1)$ & $5.5716(1)$ & $13.8426(1)$ & $372.15(1)$ \\
0.10 & $R 3 c(90 \%)$ & $5.5630(2)$ & $5.5630(2)$ & $13.8061(6)$ & $370.02(2)$ \\
& Pnma $(10 \%)$ & $5.6529(15)$ & $7.8174(32)$ & $5.4196(12)$ & $239.50(13)$ \\
0.15 & $R 3 c(51 \%)$ & $5.5614(2)$ & $5.5614(2)$ & $13.8020(8)$ & $369.69(3)$ \\
& Pnma (49\%) & $5.6272(2)$ & $7.8115(3)$ & $5.4325(2)$ & $238.80(1)$ \\
0.20 & R3c (9\%) & $5.5604(2)$ & $5.5604(2)$ & $13.7984(13)$ & $369.46(4)$ \\
& Pnma $(91 \%)$ & $5.6253(3)$ & $7.7987(4)$ & $5.4231(3)$ & $237.91(2)$ \\
0.25 & Pnma $(100 \%)$ & $5.6261(2)$ & $7.7893(3)$ & $5.4213(2)$ & $237.58(1)$ \\
0.30 & Pnma $(100 \%)$ & $5.6249(1)$ & $7.7789(1)$ & $5.4152(1)$ & $236.95(0)$ \\
\hline
\end{tabular}

* contains I23-type impurity phase 
Table S2. Refined room-temperature structural parameters for selected single phase $(R 3 c$ and Pnma) and biphasic $(R 3 c+P n m a)$ samples.

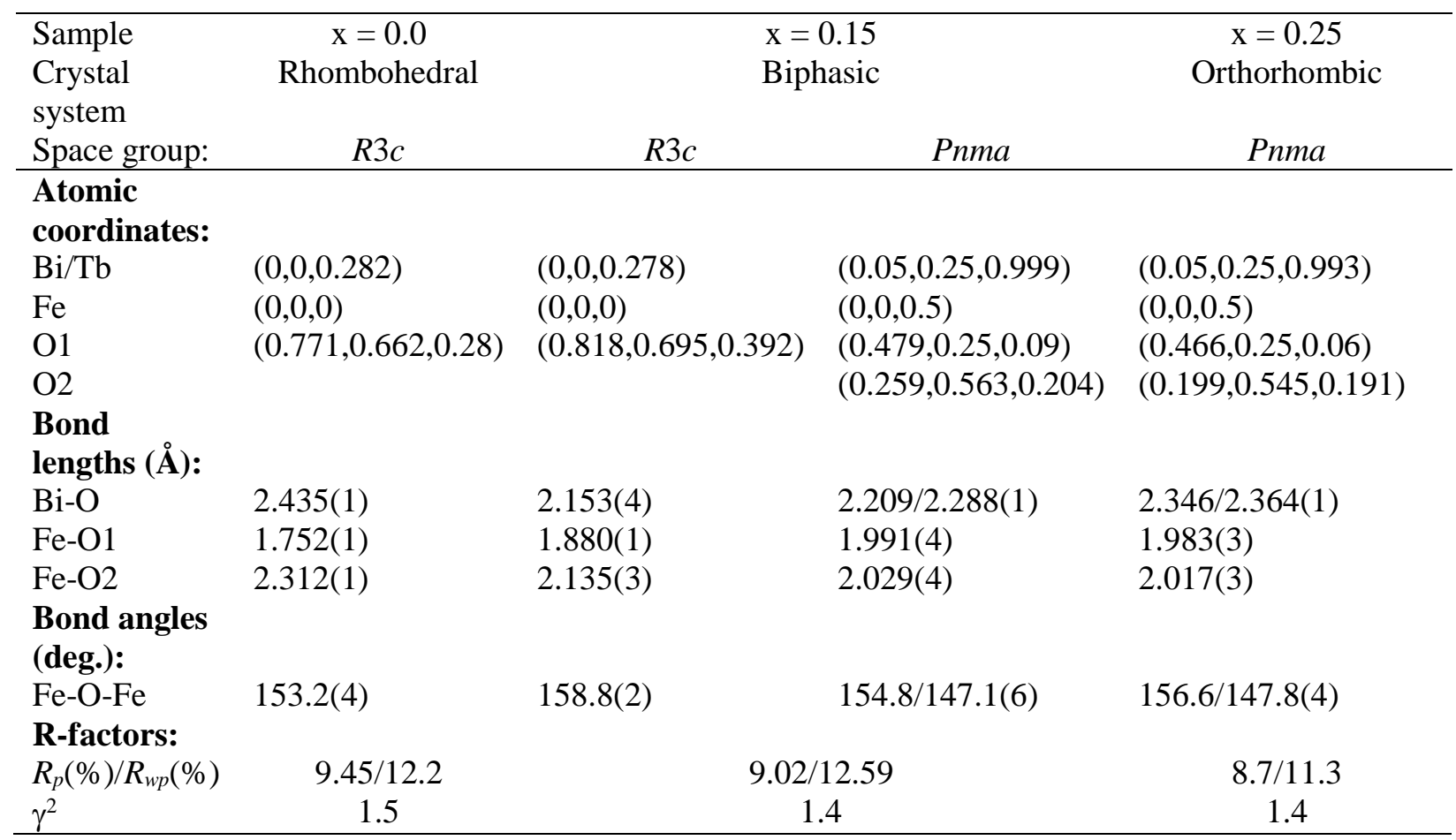

Table S3. Frequencies (in $\mathrm{cm}^{-1}$ ) and assignment of the $R 3 c$ signature Raman modes obtained for the $\mathrm{Bi}_{1-\mathrm{x}} \mathrm{Tb}_{\mathrm{x}} \mathrm{FeO}_{3}(0 \leq \mathrm{x} \leq 0.20)$ samples at room temperature.

\begin{tabular}{llllll}
\hline Mode & $\mathrm{x}=0$ & $\mathrm{x}=0.05$ & $\mathrm{x}=0.10$ & $\mathrm{x}=0.15$ & $\mathrm{x}=0.20$ \\
\hline$E$ (TO1) & 70 & 70 & 69 & 69 & 71 \\
$E$ (TO2) & 135 & 137 & 137 & 138 & 141 \\
$A_{1}(\mathrm{LO} 1)$ & 167 & 170 & 171 & 171 & 171 \\
$A_{1}(\mathrm{LO} 2)$ & 215 & 218 & 224 & 224 & 233 \\
\hline
\end{tabular}



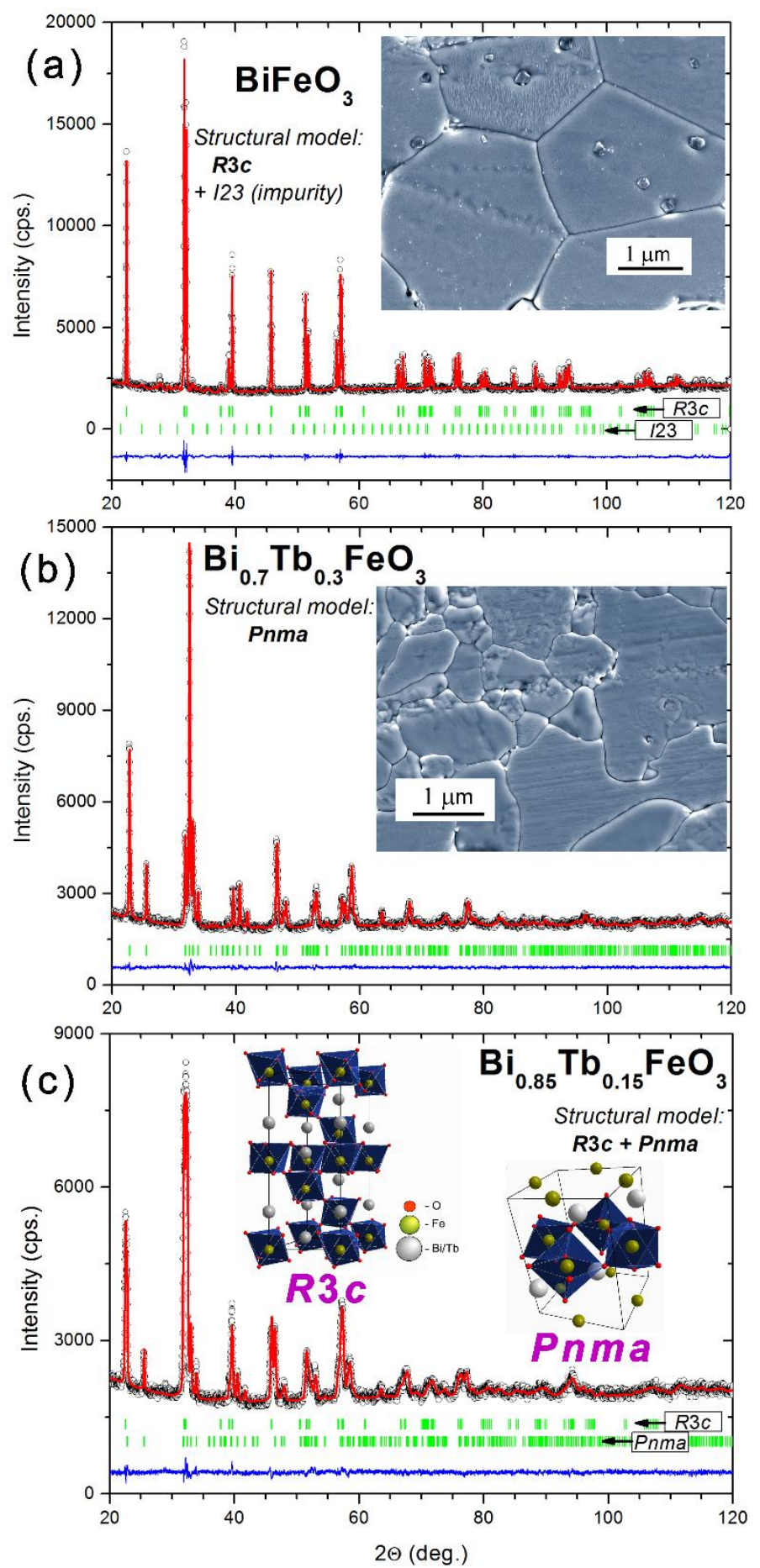

Figure S1. Observed (black open circles), calculated (red solid line) and difference (blue solid line) patterns obtained from the Rietveld analysis of the $\mathrm{X}$-ray diffraction data of $\mathrm{Bi}_{1-}$

${ }_{\mathrm{x}} \mathrm{Tb}_{\mathrm{x}} \mathrm{FeO}_{3}$ ceramics for (a) $\mathrm{x}=0$, (b) $\mathrm{x}=0.30$, and (c) $\mathrm{x}=0.15$. The allowed Bragg reflections for the corresponding space groups (R3c, Pnma and I23) are marked by green ticks. The insets in (a) and (b) show micrographs of respective compositions. The inset in (c) illustrates the schematic representations of the crystal structures coexisting in biphasic $(R 3 c+P n m a) \mathrm{x}=$ 0.15 sample. 

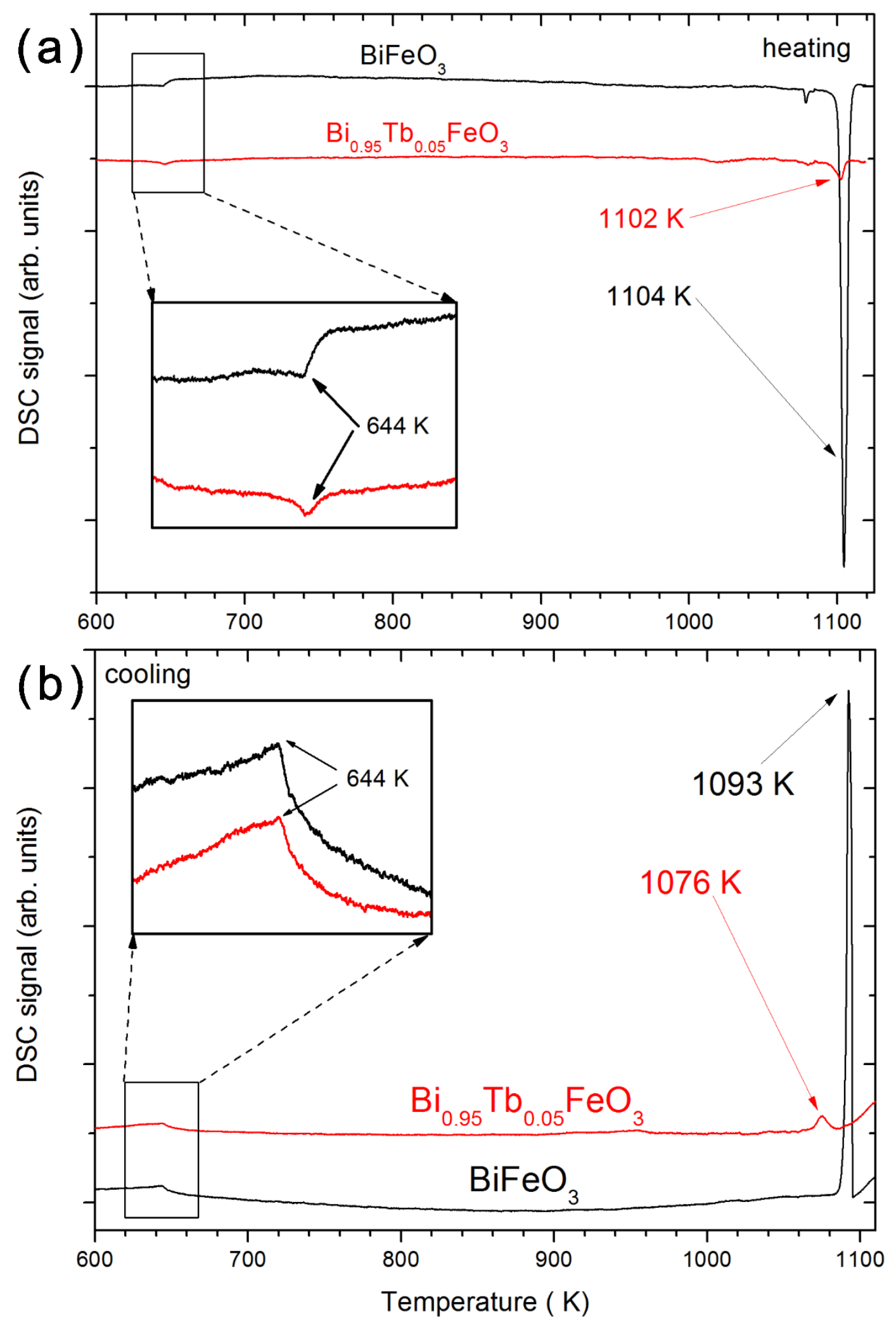

Figure S2. Comparison of the thermal behavior of the $\mathrm{BiFeO}_{3}$ and $\mathrm{Bi}_{0.95} \mathrm{~Tb}_{0.05} \mathrm{FeO}_{3}$ samples; (a) heating run, (b) cooling run. 


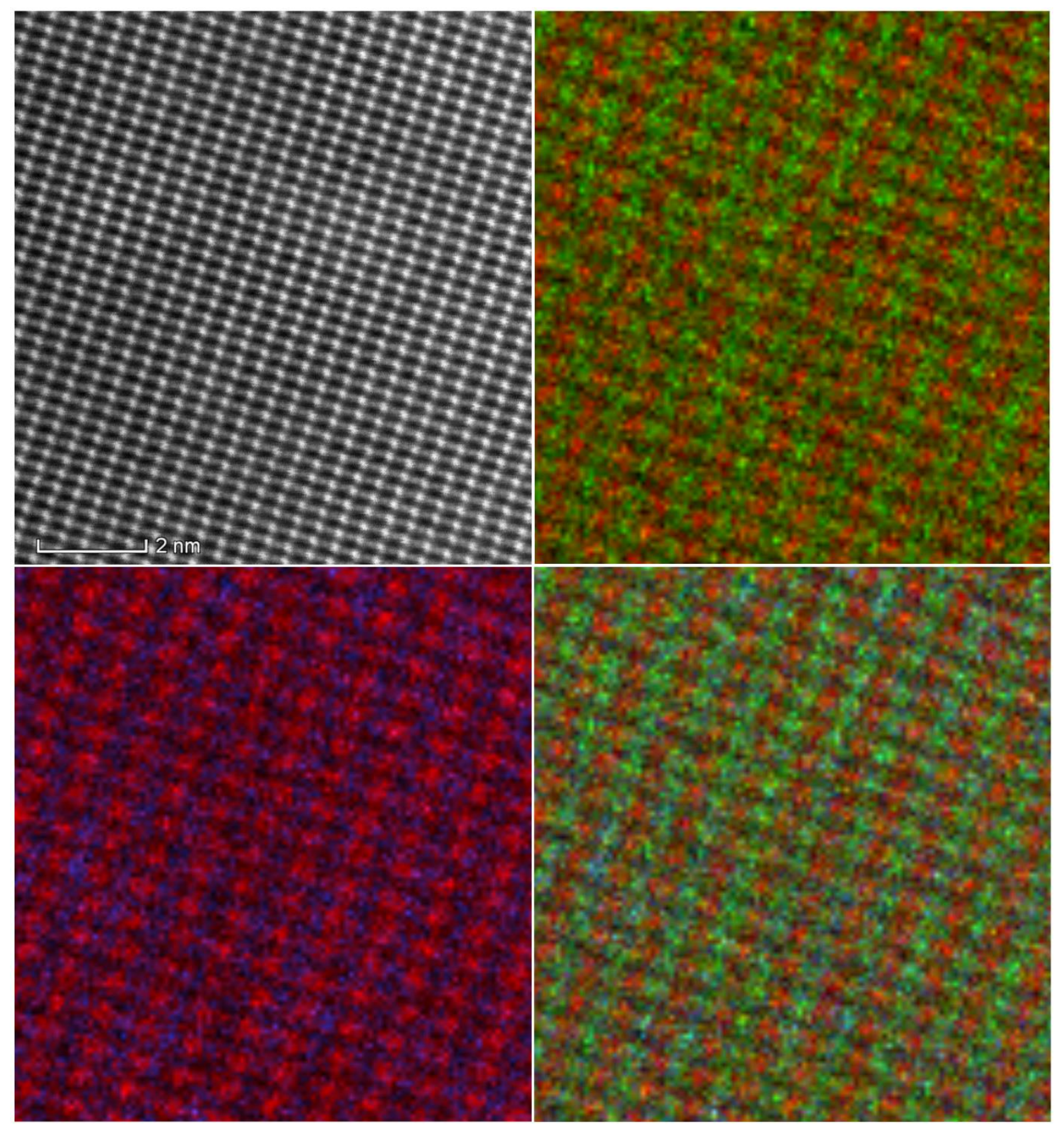

Figure S3. Two-dimensional elemental distribution maps derived from the same HAADFSTEM image (a) of $\mathrm{Bi}_{0.7} \mathrm{~Tb}_{0.3} \mathrm{FeO}_{3}$ for (b) $\mathrm{Bi}$ (green)/Fe (red), (c) $\mathrm{Tb}$ (blue)/Fe (red), and (d) composed $\mathrm{Bi}$ (green)/ $\mathrm{Tb}$ (blue)/Fe (red) to confirm the occupancy of the $\mathrm{A}$ - sites by $\mathrm{Bi}$ and $\mathrm{Tb}$ and the B- site by Fe. 


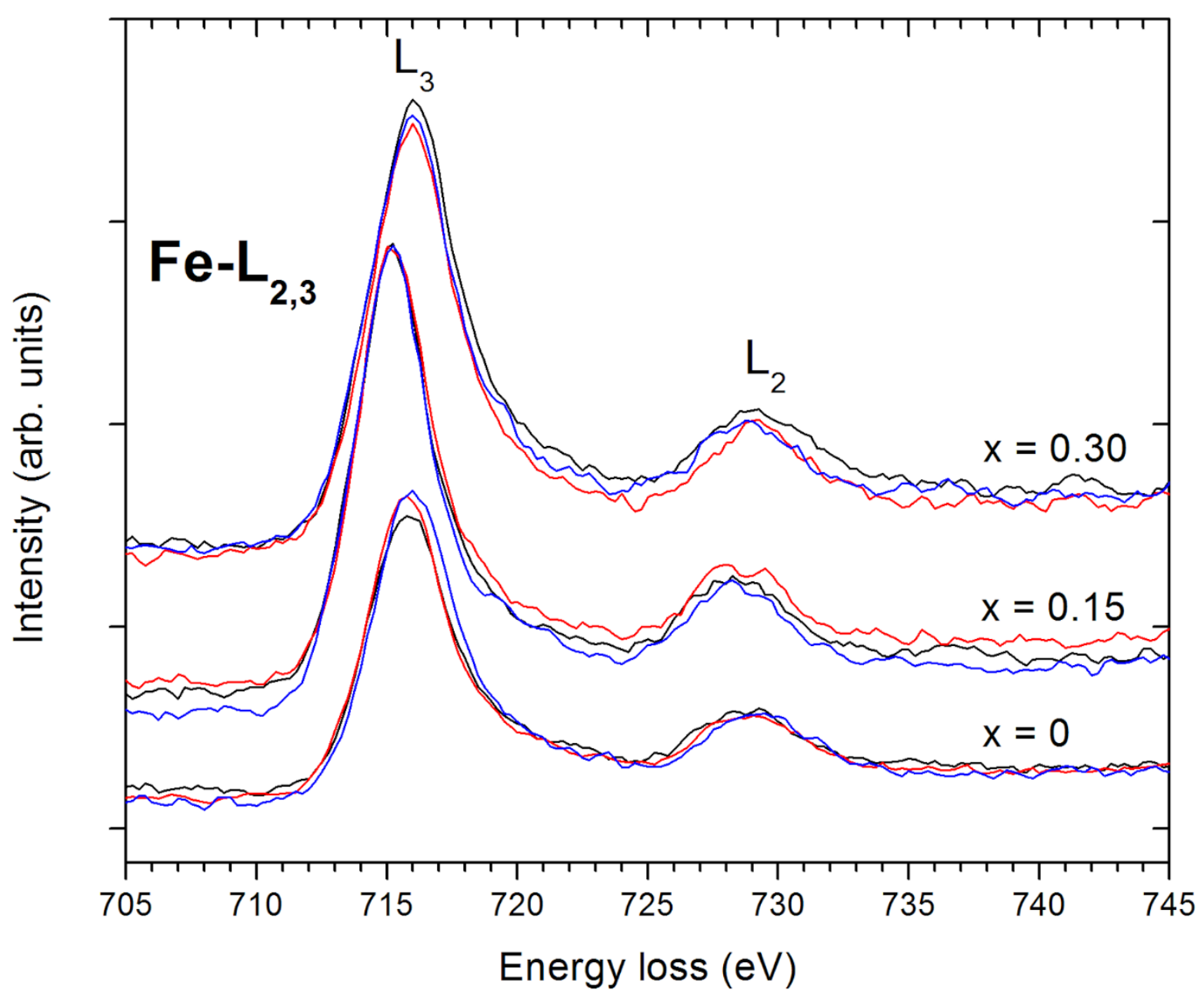

Figure S4. The ELNES spectra acquired at the $\mathrm{Fe} \mathrm{L}_{2,3}$ edge of $\mathrm{Bi}_{1-\mathrm{x}} \mathrm{Tb}_{\mathrm{x}} \mathrm{FeO}_{3}(\mathrm{x}=0,0.15$ and 0.30). The representative spectra for each composition were obtained from three different regions of the sample (scanned region $4 \times 4 \times 15 \mathrm{~nm}$ ) of nearly the same thickness and the same crystallographic orientation for the limited time of exposition of $10 \mathrm{~s}$ in order to minimize the effects of electron irradiation on the sample materials (avoid electron-beam damage of the sample material). Other regions of samples displayed almost identical spectral features at the $\mathrm{O} \mathrm{K}$ and $\mathrm{Fe}_{2,3}$ edges. 


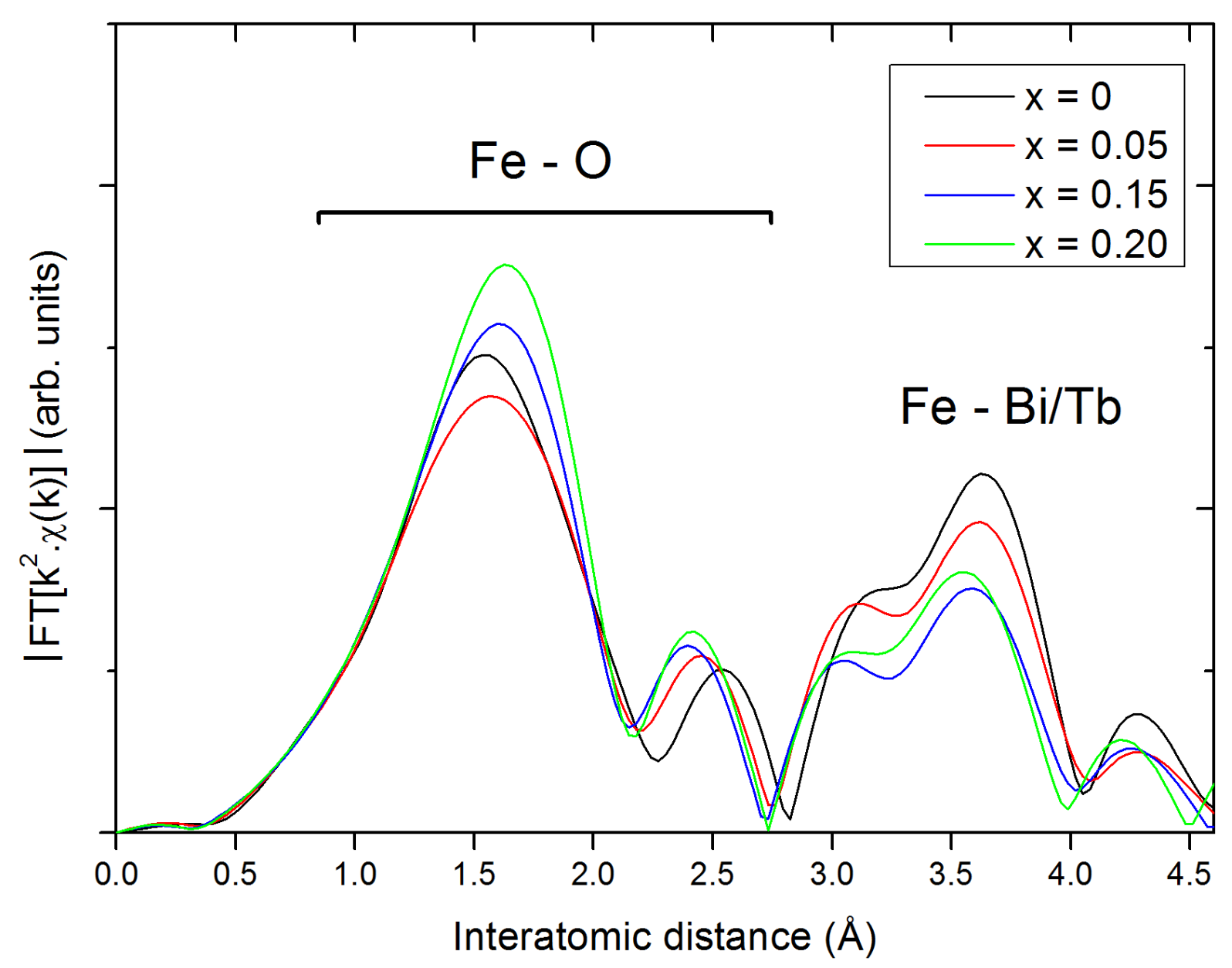

Figure S5. Fourier transformation of the EXAFS function, $\chi(\mathrm{k})$, into real space for $\mathrm{Bi}_{1-}$ ${ }_{x} \mathrm{~Tb}_{\mathrm{x}} \mathrm{FeO}_{3}(\mathrm{x}=0,0.05,0.15$ and 0.20$)$.
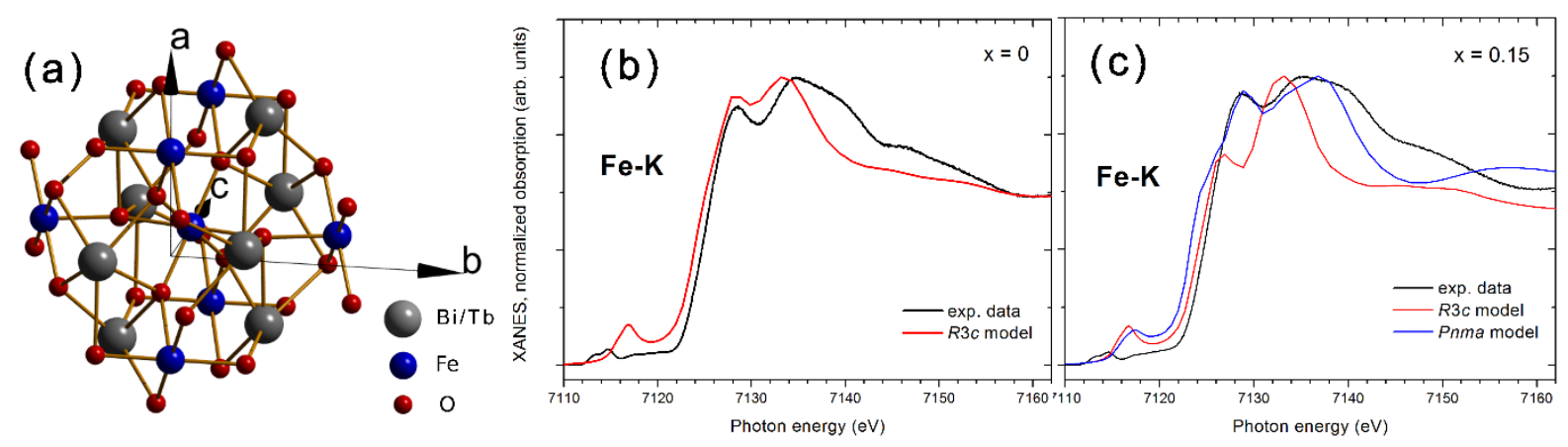

Figure S6. (a) The calculated crystallographic cluster around the absorber Fe atom $(5 \AA$ radius) from the XANES data (FEFF 8.1). (b) and (c) show the experimental and simulated XANES spectra of pure $(x=0)$ and doped $(x=0.15) \mathrm{BiFeO}_{3}$, respectively. 


\section{References}

${ }^{1}$ N. A. Hill, Journal of Physical Chemistry B, 2000, 104, 6694-6709.

${ }^{2}$ M. Fiebig, Journal of Physics D: Applied Physics, 2005, 38, R123-R152.

${ }^{3}$ R. Seshadri, N.A. Hill, Chemistry of Materials, 2001, 13, 2892-2899.

${ }^{4}$ G. Catalan, J.F. Scott, Advanced Materials, 2009, 21, 2463-2485.

${ }^{5}$ S. W. Cheong, M. Mostovoy, Nature Materials, 2007, 6, 13-20.

${ }^{6}$ J. B. Neaton, C. Ederer, U. V. Waghmare, N. A. Spaldin, K. M. Rabe, Physical Review B, 2005, 71, 014113-1-18.

${ }^{7}$ S. M. Selbach, M. -A. Einarsrud, T. Grande, Chemistry of Materials, 2009, 21,169-173.

${ }^{8}$ L. W. Martin, Dalton Transactions, 2010, 39, 10813-10826

${ }^{9}$ J. T. Wu, S. Y. Mao, Z. G. Ye, Z. Z. Xie, L. S. Zheng, Journal of Materials Chemistry, 2010, 20, 6512-6516.

${ }^{10}$ Q. Xu, M. Sobhan, Q. Yang, F. Anariba, K. P. Ong, P. Wu, Dalton Transactions, 2014, 43, 10787-10793.

${ }^{11}$ L. W. Martin, Y. H. Chu, R. Ramesh, Materials Science Engineering R, 2010, 68, 89-133.

12 J. M. Moreau, C. Michel, R. Gerson, W. J. James, Journal of Physics and Chemistry of Solids, 1971, 32, 1315-1320.

${ }^{13}$ A. Perejón, P. E. Sánchez-Jiménez, L. A. Pérez-Maqueda, J. M. Criado, J. Romero de Paz, R. Sáez-Puche, N. Masó, A. R. West, Journal of Matererials Chemistry C, 2014, 2, 83988411.

${ }^{14}$ Q. Jiang, H. Ning, Q. Zhang, M. Cain, M. J. Reece, H. Yan, Journal of Materials Chemistry $C, 2013,1,5628-5631$.

${ }^{15}$ V. A. Khomchenko, D. A. Kiselev, M. Kopcewicz, M. Maglione, V. V. Shvartsman, P. Borisov, W. Kleemann, A. M. L. Lopes, J. P. Araujo, R. M. Rubinger, N. A. Sobolev, A. L. Kholkin, Journal of Magnetism and Magnetic Materials, 2009, 321, 1692-1698.

${ }^{16}$ S. M. Selbach, T. Tybell, M. A. Einarsrud, T. Grande, Chemistry of Materials, 2009, 21, 5176-5186.

${ }^{17}$ V. Koval, I. Skorvanek, M. Reece, L. Mitoseriu, H. Yan, Journal of the European Ceramic Society, 2014, 34, 641-651.

${ }^{18}$ I. O. Troyanchuk, D. V. Karpinsky, M. V. Bushinsky, O. S. Mantytskaya, N. V. Teresko, V. N. Shut, Journal of the American Ceramic Society, 2011, 94, 4502-4506.

${ }^{19}$ A. R. Akbashev, A. S. Semisalova, N. S. Perov, A. R. Kaul, Applied Physics Letters, 2011, 99, 122502-122505.

${ }^{20}$ D. Kan, L. Palova, V. Anbusathaiah, C. J. Cheng, S. Fujino, V. Nagarajan, K. M. Rabe, I.

Takeuchi, Advanced Functional Materials, 2010, 20, 1108-1115.

${ }^{21}$ M. S. Sverre, E. M. Ann, G. Torr, Chemistry of Materials, 2009, 21, 169-173.

${ }^{22}$ R. D. Shannon, Acta Crystallograffica A, 1976, 32, 751-767.

${ }^{23}$ Y. Q. Jia, Journal of Solid State Chemistry, 1991, 95, 184-187.

${ }^{24}$ Y. Cao, M. Xiang, W. Zhao, G. Wang, Z. Feng, B. Kang, A. Stroppa, J. Zhang, W. Ren, S. Cao, Journal of Applied Physics, 2016, 119, 063904-1-6.

${ }^{25}$ Q. Jiang, Y. Lin, C. -W. Nan, Journal of the American Ceramic Society, 2007, 90, 14441447.

${ }^{26}$ Y. -J. Zhang, H. -G. Zhang, J. -H. Yin, H. -W. Zhang, J. -L. Chen, W. -Q. Wang, G. -H, Journal of Magnetism and Magnetic Materials, 2010, 322, 2251-2255.

${ }^{27}$ S. Saxin, C. S. Knee, Journal of Solid State Chemistry, 2011, 184, 1576-1579.

${ }^{28}$ V. Koval, I. Skorvanek, H. Yan, Journal of the American Ceramic Society, 2014, 97, 37293732.

${ }^{29}$ J. Bielecki, P. Svedlindh, D. T. Tibebu, S. Cai, S. -G. Eriksson, L. Borjesson, C. S. Knee, Physical Review B, 2012, 86, 184422-1-16. 
${ }^{30}$ G. S. Lotey, N. K. Verma, Journal of Nanoparticles Research, 2013, 15, 1553-1-14.

${ }^{31}$ G. Dhir, G. S. Lotey, P. Uniyal, N. K. Verma, Journal of Material Science: Materials in Electronics, 2013, 24, 4386-4392.

${ }^{32}$ J. Zhang, Y. -J. Wu, X. -K. Chen, X. -J. Chen, Journal of Physicsm and Chemistry of Solids, 2013, 74, 849-853.

${ }^{33}$ Z. -H. Dai, Y. Akishige, Ceramics International, 2012, 38, S403-S406.

${ }^{34}$ J. Rodriguez-Carvajal, Physica B, 1993, 192, 55-69.

${ }^{35}$ S. Karimi, I. Reaney, I. Levin, I. Sterianou, Applied Physics Letters, 2009, 947, 112903-13.

${ }^{36}$ E. Okunishi, H. Sawada, Y. Kondo, Micron, 2012, 43, 538-544.

${ }^{37}$ E. Okunishi, I. Ishikawa, H. Sawada, F. Hosokawa, M. Hori, Y. Kondo, Microscopy and Microanalysis, 2009, 15, 164-165.

${ }^{38}$ C. Gauthier, V. A. Sole', R. Signorato, J. Goulon, E. Moguiline, Journal of Synchrotron Radiation, 1999, 6, 164-166.

${ }^{39}$ A. L. Ankudinov, B. Ravel, J. J. Rehr, S. D. Conradson, Physical Reviev B, 1998, 58, 7565.

${ }^{40}$ K. V. Klementev, Journal of Physics D: Applied Physics, 2001, 34, 209-217.

${ }^{41}$ M. Muneeswaran, R. Dhanalakshmi, N. V. Giridharan, Journal of Material Science: Materials in Electronics, 2015, 26, 3827-3839.

${ }^{42}$ M. -C. Guo, W. -F. Liu, P. Wu, H. Zhang, X. -L. Xu, S. -Y. Wang, G. -H. Rao, Chinese Physics Letters, 2015, 32, 066101-1-5.

${ }^{43}$ P. C. Sati, M. Kumar, S. Chhoker, Ceramics International, 2015, 41, 3227-3236.

${ }^{44}$ Y. J. Yoo, J. S. Hwang, Y. P. Lee, J. S. Park, J. Y. Rhee, J. -H. Kang, K. W. Lee, B. W. Lee, M. S. Seo, Journal of Magnetism and Magnetic Materials, 2015, 374, 669-675.

${ }^{45}$ Crystallography Open Database, http://www.crystallography.net

${ }^{46}$ G. D. Achenbach, W. J. James, R. Gerson, Journal of the American Ceramic Society, 1967, 50, 437.

${ }^{47}$ A. M. L. Lopes, J. P. Araújo, S. Fedorov, Dalton Transactions, 2014, 43, 18010-18016.

${ }^{48}$ Q. Zhang, X. Zhu, Y. Xu, H. Gao, Y. Xiao, D. Liang, J. Zhu, D. Xiao, Journal of Alloys and Compounds, 2013, 546, 57-62.

${ }^{49}$ L. R. Guo, W. Fang F. Dong, Z. M. Shen, Journal of Physical Chemistry C, 2010, 114, 21390-21396.

${ }^{50}$ J. -B. Li, G. H. Rao, Y. Xiao, J. K. Liang, J. Luo, G. Y. Liu, J. R. Chen, Acta Materialia, 2010, 58, 3701-3708.

${ }^{51}$ P. Fischer, M. Polomska, I. Sosnowska, M. Szymanski, Journal of Physics C: Solid State Physics, 1980, 13, 1931-1940.

52 A. Jaiswal, R. Das, K. Vivekanand, P. M. Abraham, S. Adyanthaya, P. Poddar, Journal of Physical Chemistry C, 2010, 114, 2108-2115.

${ }^{53}$ M. Marezio, J. P. Remeika, P. D. Dernier, Acta Crystallographica B, 1970, 26, 2008-2022.

${ }^{54}$ A. M.Glazer, Acta Crystallograpfica A, 1975, 31, 756-762.

${ }^{55}$ V. A. Khomchenko, J. A. Paixao, V. V. Shvartsman, P. Borisov, W. Kleeman, D. V. Karpinsky, A. L. Kholkin, Scripta Materialia, 2010, 62, 238-241.

${ }^{56}$ I. O. Troyanchuk, M. V. Bushinsky, D. V. Karpinsky, O. S. Mantytskaya, V. V. Fedotova, O. I. Prochnenko, Physica Status Solidi B, 2009, 246, 1901-1907.

${ }^{57}$ O. Guillon, J. Gonzalez-Julian, B. Dargatz, T. Kessel, G. Schierning, J. Rathel, M.

Herrman, Advanced Engineering Materials, 2014, 16, 830-849.

${ }^{58}$ P. Hermet, M. Goffinet, J. Kreisel, Ph. Ghosez, Physical Review B, 2007, 75, 220102-14(R).

${ }^{59}$ J. Hlinka, J. Pokorny, S. Karimi, I.M. Reaney, Physical Review B, 2011, 83, 020101-14(R).

${ }^{60}$ G. L. Yuan, S. W. Or, H. L. W. Chan, Journal of Applied Physics, 2007, 101, 064101-1-5. 
${ }^{61}$ M. N. Iliev, M. V. Abrashev, H. -G. Lee, V. N. Popov, Y. Y. Sun, C. Thomsen, R. L. Meng, C. W. Chu, Physical Review B, 1998, 54, 2872-2877.

${ }^{62}$ I. Sosnowska, T. Peterlin-Neumaier, E. Steichele, Journal of Physics C: Solid State Physics, 1982, 15, 4835-4846.

63 J. Walker, H. Ursic, A. Bencan, B. Malic, H. Simons, I. Reaney, G. Viola,V. Nagarajan, T. Rojac, Journal of Materials Chemistry C, 2016, 4, 7859-7868.

${ }^{64}$ T. Rojac, M. Kosec, B. Budic, N. Setter, D. Damjanovic, Journal of Applied Physics, 2010, 108, 074107-1-8.

${ }^{65}$ I. Sosnowska, W. Schafer, W. Kockelman, K.H. Andersen, I.O. Troyanchuk, Applied Physics Letters, 2002, 74, 1040-2.

${ }^{66}$ A. V. Zalesskii, A. A. Frolov, T. A. Khimich, A. A. Bush, Physics of the Solid State, 2003, 45, 141-145.

${ }^{67}$ V. R. Reddy, D. Kothari, A. Gupta, S. M. Gupta, Applied Physics Letters, 2009, 94, 082505-1-3.

${ }^{68}$ P. Modak, D. Lahiri, S. M. Sharma, Journal of Physical Chemistry C, 2016, 120, 84118416.

${ }^{69}$ Z. Y. Wu, S. Gota, F. Jollet, M. Pollak, M. Gautier-Soyer, C. R. Natoli, Physical Review B, 1997, 55, 2570-2577.

${ }^{70}$ R. Saeterli, S. M. Selbach, P. Ravindran, T. Grande, R. Holmestad, Physical Review B, 2010, 82, 064102.

${ }^{71}$ P. S. Sankara Rama Krishnan, J. A. Aguiar, Q. M. Ramasse, D. M. Kepaptsoglou, W. -I. Liang, Y. -H. Chu, N. D. Browning, P. Munroe, V. Nagarajan, Journal of Materials Chemistry C, 2015, 3, 1835-1845.

${ }^{72}$ T. J. Park, S. Sambavisan, D. A. Fischer, W.S. Yoon, J. A. Misewich, S. S. Wong, Journal of Physical Chemistry C, 2008, 112, 10359-10369.

${ }^{73}$ T. Higuchi, Y. -S. Liu, P. Yao, P. -A. Glans, J. Guo, C. Chang, Z. Wu, W. Sakamoto, N. Itoh, T. Shimura, T. Yogo, T. Hattori, Physical Review B, 2008, 78, 085106.

${ }^{74}$ P. Ravindran, R. Vidya, A. Kjekshus, H. Fjellvåg, O. Eriksson, Physical Review B, 2006, 74, 224412.

${ }^{75}$ M. Abbate, F. M. F. de Groot, J. C. Fuggle, A. Fujimori, O. Strebel, F. Lopez, M. Domke, G. Kaindl, G. A. Sawatzky, M. Takano, Y. Takeda, H. Eisaki, S. Uchida, Physical Review B, 1992, 46, 4511.

${ }^{76}$ F. M. F. de Groot, M. Grioni, J. C. Fuggle, J. Ghijsen, G. A. Sawatzky, H. Petersen, Physical Review B, 1989, 40, 5715-5723.

77 Z. L. Wang, J. S. Yin, Y. D. Jiang, Micron, 2000, 31, 571-580.

${ }^{78}$ P. A. van Aken, B. Liebscher, Physics and Chemistry of Minerals, 2002, 29, 188-200.

${ }^{79}$ R. Leapman, L. Grunes, Physical Reviev Letters, 1980, 45, 397-401.

${ }^{80}$ S. - Y. Chen, A. Gloter, A. Zobelli, L. Wang, C. -H. Chen, C. Colliex, Physical Review B, 2009, 79, 104103-1-10.

${ }^{81}$ C. Colliex, T. Manoubi, C. Ortiz, Physical Review B, 1991, 44, 11402-11411.

${ }^{82}$ A. Y. Borisevich, E. A. Eliseev, A. N. Morozovska, C. -J. Cheng, J. -Y. Lin, Y. H. Chu, D. Kan, I. Takeuchi, V. Nagarajan, S. V. Kalinin, Nature Communications, 2012, 3, 775.

${ }^{83}$ L. A. Grunes, Physical Review B, 1983, 27, 2111-2131.

${ }^{84}$ T. E. Westre, P. Kennepohl, J. G. DeWitt, B. Hedman, K. O. Hodgson, E. I. Solomon, Journal of the American Ceramic Society, 1997, 119, 6297-6314.

${ }^{85}$ J. -H. Lee, H. J. Choi, D. Lee, M. G. Kim, C. W. Bark, S. Ryu, M. -A. Oak, H. M. Jang, Physical Review B, 2010, 82, 045113-1-8.

${ }^{86}$ H. Wen, M. Sassi, Z. Luo, C. Adamo, D. G. Schlom, K. M. Rosso, X. Zhang, Scientific Reports, 2005, 5, 15098. 
${ }^{87}$ Y. Yoneda, Y. Kitanaka, Y. Noguchi, M. Miyayama, Physical Review B, 2012, 86, 184112$1-11$.

${ }^{88}$ J. Jutimoosik, S. Hunpratub, S. Maensiri, S. Rujirawat, R. Yimnirun, Journal of Applied Physics, 2014, 116, 104105-1-4.

${ }^{89}$ K. -T. Ko, M. H. Jung, Q. He, J. H. Lee, C. S. Woo, K. Chu, J. Seidel, B. -G. Jeon, Y. S. Oh, K. H. Kim, W. -I. Liang, H. -J. Chen, Y. -H. Chu, Y. H. Jeong, R. Ramesh, J. -H. Park, C. -H. Yang, Nature Communications, 2011, 2, 567.

${ }^{90}$ J. F. Ihlefeld, N. J. Podraza, Z. K. Liu, R. C. Rai, X. Xu, T. Heeg, Y. B. Chen, J. Li, R. W. Collins, J. L. Musfeldt, X. Q. Pan, J. Schubert, R. Ramesh, and D. G. Schlom, Applied Physics Letters, 2008, 92, 142908.

${ }^{91}$ C. -S. Tu, C. -S. Chenb, P. -Y. Chenc, H. -H. Weia, V. H. Schmidtd, C. -Y. Lina, J. Anthoniappene, J. -M. Leef, Journal of the European Ceramic Society, 2016, 36, 1149-1157.

${ }^{92}$ D. Lee, M. G. Kim, S. Ryu, H. M. Jang, S. G. Lee, Applied Physics Letters, 2005, 86, 222903-1-3.

${ }^{93}$ S. Kripasindhu, J. Hong, G. Catalan, P. K. Biswas, M. R. Lees, R. I. Walton, J. F. Scott, S. A. T. Redfern, Journal of Physics: Condensed Matter, 2012, 24, 045905-1-8.

${ }^{94}$ Y. -K. Jun, S. B. Lee, M. Kim, S. -H. Hong, J. W. Kim, K. H. Kim, Journal of Materials Research, 2007, 22, 3397-3403.

${ }^{95}$ F. M. F. de Groot, J. Faber, J. J. M. Michiels, M. T. Czyżyk, M. Abbate, J. C. Fuggle, Physical Review B, 1993, 48, 2074-2080.

${ }^{96}$ M. -H. Whangbo, H. -J. Koo, A. Villesuzanne, M. Pouchard, Inorganic Chemistry, 2002, 41, 1920-1929.

${ }^{97}$ C. Ederer, N. A. Spaldin, Physical Review B, 2005, 71, 060401R.

${ }^{98}$ S. V. Kiselev, R. P. Ozerov, G. S.Zhdanov, Soviet Physics-Doklady, 1963, 7, 742-744.

${ }^{99}$ I. Sosnowska, M. Loewenhaupt, W. I. F. David, R. M. Ibberson, Materrials Science Forum, 1993, 133-136, 683-686.

${ }^{100}$ B. Ruette, S. Zvyagin, A. P. Pyatakov, A. Bush, J. F. Li, V. I. Belotelov, A. K. Zvezdin, D. Viehland, Physical Review B, 2004, 69, 064114.

${ }^{101}$ C. -H Yang, D. Kan, I. Takeuchi, V. Nagarajan, J. Seidel, Physical Chemistry Chemical Physics, 2012, 14, 15953-15962.

102 P. Trivedi, S. Katba, S. Jethva, M. Udeshi, B. Vyas, M. Vagadia, S. Gautam, K.H. Chae, K. Asokan, D. G. Kuberkarl, Solid State Communications, 2015, 222, 5-8.

${ }^{103}$ M. Pugaczowa-Michalska, J. Kaczkowski, Journal of Materials Science, 2015, 50, 62276235.

${ }^{104}$ N. Wang, J. Cheng, A. Pyatakov, A. K. Zvezdin, J. F. Li, L. E. Cross, D. Viehland, Physical Review B, 2005, 72, 104434-1-5.

${ }^{105}$ T.-J. Park, G. C. Papaefthymiu, A. J. Viescas, Y. Lee, H. Zhou, S. S. Wong, Physical Review B, 2010, 82, 024431-1-10.

${ }^{106}$ B. Ahmmad, M. Z. Islam, A. Billah, M. A. Basith, Journal of Physics D: Applied Physics, 2016, 49, 095001-1-7.

${ }^{107}$ D. Lebeugle, D. Colson, A. Forget, M. Viret, P. Bonville, F. Marucco, S. Fusil, Physical Review B, 2007, 76, 024116-1-8.

${ }^{108}$ N. Shamir, E. Gurewitz, H. Shaked, Acta Crystallographica A, 1978, 34, 662-666.

${ }^{109}$ M. K. Singh, W. Prellier, M. P. Singh, R. S. Katiyar, J. F. Scott, Physical Review B, 2008, 77, 144403-1-5.

110 T. -J. Park, G. C. Papaefthymiu, A. J. Viescas, A. R. Moodenbaugh, S. Wong, Nano Letters, 2007, 7, 766-772.

${ }^{111}$ K. H. J. Buschow, Handbook of Magnetic Materials. Vol. 21, North-Holland, Elsevier B. V., 2013. 